\title{
Plants of the American continent with antimalarial activity
}

\author{
Ingrid R. Mariath, Heloina de S. Falcão, José M. Barbosa-Filho*, Layanna C. F. de Sousa, \\ Anna Cláudia de A. Tomaz, Leônia M. Batista, Margareth de Fátima F. M. Diniz, Petrônio F. \\ Athayde-Filho, Josean Fechine Tavares, Marcelo S. Silva, Emídio Vasconcelos L. da Cunha
}

Laboratório de Tecnologia Farmacêutica, Universidade Federal da Paraíba, Caixa Postal 5009, 58051-900 João Pessoa, PB, Brazil

\begin{abstract}
RESUMO: "Plantas do continente Americano com atividade antimalárica". Malária é uma doença parasitária humana causada por protozoários do gênero Plasmodium. Esta doença tem acometido populações que habitam regiões tropicais e subtropicais. Anualmente, cerca de 500 milhões de casos ocorrem no mundo, o que permite ser considerada uma doença emergente de importância para a saúde pública. Neste contexto, os produtos naturais, a exemplo das espécies vegetais, têm suas moléculas bioativas como alvo para estudos farmacológicos, toxicológicos e fitoquímicos destinados à síntese de medicamentos mais eficazes para o tratamento de inúmeras doenças. Portanto, este trabalho fornece subsídio às pesquisas com produtos naturais para o tratamento da malária. Nesta revisão, 476 espécies de plantas do continente Americano foram relatadas para a atividade antimalárica, sendo destas 198 ativas e 278 inativas para algum tipo de Plasmodium, quando avaliados através de modelos in vitro e in vivo.
\end{abstract}

Unitermos: Atividade antimalárica, plantas medicinais, produtos naturais, continente Americano, revisão.

\begin{abstract}
Malaria is a human parasitic disease caused by protozoa species of the Plasmodium genus. This disease has affected populations of the tropical and subtropical regions. About 500 million new cases occur annually on the world and therefore it is considered an emerging disease of important public health problem. In this context, the natural products as vegetables species have their bioactive molecules as targets for pharmacological, toxicological and phytochemical studies towards the development of more effective medicines for the treatment of many diseases. So this work intends to aid the researchers in the study of natural products to the treatment of malaria. In this review, 476 plants of the American continent were related for the antimalarial activity and of these vegetables species 198 were active and 278 inactive for some type of Plasmodium when they were evaluated through of in vitro or in vivo bioassays models.
\end{abstract}

Keywords: Antimalarial activity, medicinal plants, natural products, American continent, review.

\section{INTRODUCTION}

Malaria is a parasitic disease caused by protozoa species of the Plasmodium genus and it is transmitted for human through the bite of infected female mosquito of the Anopheles genus (Veronesi, 1991). In each year, about 100 - 500 million people are infected while 1 - 3 million died with malaria in the world. This disease is considered a public health problem of global scale by World Health Organization (WHO, 1997). It is estimated that $40 \%$ of the population world lives in areas at risk of infection over 100 countries worldwide, which are include countries of the American continent (Trigger \& Kondrachine, 1998).

In the South America, especially in Bolivia, the malaria is responsible for considerable number of morbidity and mortality. According to the Organización Panamericana de la Salud (OPS 1997), 0.7\% of the Argentine population lives at risk of infection, but this percentage could still be higher because cross-border migration and the unrelated cases in the rural areas (Debenedetti et al., 2002). In Colombia, it was registered about 120,000 cases of malaria in the decade of 90 , while in Brazil, 509.000 cases were recorded in this same period with $96 \%$ of them occurred in the Amazon region (WHO, 1997).

The main species of the genus Plasmodium involved on the transmition of the malaria for human are: $P$. vivax, $P$. ovale, $P$. falciparum and $P$. malaria. Therefore, the third specie is the most causer of death (Harrison et al., 1998). Other species of this genus infect animals as P. knowlesi (monkeys), P. berghei (rodents) and $P$. gallinaceum (fowl). The clinical events are fever, feeling of malaise, chills, muscle pain, fatigue, anemia, compromised liver, spleen and kidney and they occur between 9 and 40 days after the bite of the infected mosquitoes, depending on the species of Plasmodium (Neves et al., 2005). 
The treatment with single or combined drugs resistant to chloroquine has been used as an alternative therapy for malaria, but they are expensive and sometimes cause signal of toxicity due to limited knowledge about the metabolism and mechanism of action of these antimalarial drugs. However, all these medicines are still are essential to reduce the morbidity and mortality caused by malaria, since the vaccine against this disease have limited activity in humans. Therefore, it is necessary to develop new, safe and effective antimalarial medicines. So in recent years, there has also been growing interest in alternative therapies such the use of natural products, especially those derived from plants which have showed to produce promising results for the treatment of many diseases (Rates, 2001). In this context, the present work is a review about plants of the American continent with antimalarial activity.

In the course of our continuing search for bioactive natural products from plants, we have recently published reviews of extracts and compounds derived of plants with the following potential activities: inhibitors of mammary, uterine cervical and ovarian neoplasia (Moura et al., 2001; Moura et al., 2002; Silva et al., 2003); inhibitors of HMG CoA reductase (Gonçalves et al., 2000); with central analgesic activity (Almeida et al., 2001); employed in prevention of osteoporosis (Pereira et al., 2002); for the treatment of Parkinson's disease (Morais et al., 2003); anticonvulsant and anxiety disorders (Quintans-Junior, 2008; Sousa et al., 2008), with antileishmanial (Rocha et al., 2005), giardicidal (Amaral et al., 2006), antileprotic (Barbosa-Filho et al., 2007a), hypoglycemic (Barbosa-Filho et al., 2005) and antiinflammatory (Falcão et al., 2005; Barbosa-Filho et al., 2006a) activities; inhibitors of the acetylcholinesterase and angiotensin-converting enzyme (Barbosa-Filho et al., 2006b; Barbosa-Filho et al., 2006c), and with antiulcer activity (Falcão et al., 2008a,b; Mota et al., 2009). Our group has also reviewed the medicinal and poisonous plants of the Northeastern region of Brazil (Agra et al., 2007, 2008), among other review articles (Alves et al., 2000; Souza et al., 2005; Gonçalves et al., 2006; BarbosaFilho et al., 2007b, 2008; Sena-Filho et al., 2008).

\section{MATERIALS AND METHODS}

In this article, some reports about vegetable species of the American continent with antimalarial activity were reviewed in the specialized literature published up to December 2007. The search was carried out on data banks such as Biological Abstracts, SciFinder Scholar, Periódicos CAPES, Pubmed and NAPRALERT (acronym for Natural Products ALERT - University of Illinois in Chicago, U.S.A.). The references were consulted for details of the experimental models used for testing the extracts and derivates vegetables against malaria, activities and organism tested.

\section{RESULTS AND DISCUSSION}

For this review, 476 species of plants were listed with possible antimalarial activity and distributed in 103 botanical families. Among those plant species 198 were active and 278 inactive against some Plasmodium type causer of malaria when evaluated in vivo or in vitro bioassays models. The antimalarial activity was evaluated for $P$. falciparum, $P$. berghei, $P$. gallinaceum, $P$. vinckei, $P$. lophurae, $P$. cathemerium and $P$. yolii stains. The most studied botanical families were Asteraceae, Simaroubaceae, Fabaceae, Meliaceae, Amaryllidaceae, Apocynaceae, Rubiaceae, Velloziaceae and Verbenaceae which showed more than ten species of plants studied. The main studies were developed in Brazil and United States due to the flora biodiversity offered by the first country or the financial-technology resource of the second one. All data are shown in Table 1.

Some researches are validation of the tradicional medicine with the use of plants for the treatment of malaria. Remijia ferruginea (Rubiaceae) of which the $80 \%$ ethanol extract obtained of the dried bark at the dose of $500 \mathrm{mg} / \mathrm{kg}$ was active against $P$. berghei infected mice (Andrade-Neto et al., 2003) and Bidens pilosa (Asteraceae), one plant used in the Brazilian endemic area, showed antimalarial activity to different extracts obtained of dried entire plant, leaves, root or stem against $P$. falciparum and $P$. berguei through of in vitro and in vivo bioassays models (Brandão et al., 1997; Krettli et al., 2001a,b; Oliveira et al., 2004). Other species of the Asteraceae family is Vernonia brasiliana in which the hexane extract of dried leaves decreased the infection of $P$. berghei in mice and it was inactive for P. falciparum in vitro model (Carvalho \& Krettli, 1991; De Almeida Alves et al., 1997); the decoction and aqueous extract of Acanthospermum australe inhibited in vitro the growth of P. falciparum stain or decreased the infection caused by $P$. berghei in mice (Brandão et al., 1992; Carvalho \& Krettli, 1991; Carvalho et al., 1991). Species of the Artemisia genus also were studied for antimalarial activity such A. absinthium, A. vulgaris and A. ludoviciana. In the researches, the aqueous extract of the first and second plant did not inhibit in vitro the growth of $P$. falciparum (Hernandez et al., 1990), while the $95 \%$ ethanol extract of the last specie was active against $P$. yoelii infected mice (Malagon et al., 1997).

The vegetables species of the Cucurbitaceae family were also evaluated to this activity. So the $95 \%$ ethanol extract obtained from the dried seed of Cucurbita maxima popularly known as pumpkin strawberries was active in dose of $250 \mathrm{mg} / \mathrm{kg}$ against $P$. berghei infected mice (Amorim et al., 1991) and Momordica charantia, called as melon from São Caetano, had the aqueous and chloroform extracts obtained of the dried flowers or aerial parts inactive against $P$. berghei, $P$. gallinaceum, P. cathemerium and P. lophurae (Carvalho et al., 1991; Spencer et al., 1947), the $95 \%$ ethanol extract obtained 
of the dried leaves in the dose of $500 \mathrm{mg} / \mathrm{kg}$ was also inactive against $P$. berghei infected mice (Amorim et al., 1991), while the infusion and $95 \%$ ethanol extract of the dried leaves and stem in dose of $1.0 \mathrm{~g} / \mathrm{kg}$ were active against $P$. berghei infected human or mice (Ueno et al., 1996).

Lantana cujabensis Schauer (Verbenaceae) is a shrub found in the Amazonian and Andean forests of South America. Okunade \& Lewis (2004) related that the $95 \%$ ethanol extract obtained of the dried leaves and stem of this plant had antimalarial activity in vitro against $P$. falciparum. Mcphail et al. (2007) evaluated the antimalarial activity of the ethyl acetate extract of marine cyanobacterium Lyngbya majuscula (Oscillatoriaceae) in vitro model for $P$. falciparum. This extract in the concentration of $2.0 \mu \mathrm{g} / \mathrm{mL}$ inhibited the growth of the protozoa.

Plant species of the Fabaceae family were also evaluated such Bowdichia virgilioides which is traditionally used against malaria by American natives had the $95 \%$ ethanol extract obtained of the dried bark active in dose of $250 \mathrm{mg} / \mathrm{kg}$ to $P$. berghei infected mice and in vitro to $P$. falciparum - MRC $20\left(\mathrm{IC}_{50}=1 \mu \mathrm{g} / \mathrm{mL}\right)$ and P. berghei $\left(\mathrm{IC}_{50}=0.2 \mu \mathrm{g} / \mathrm{mL}\right)$. In similar, Caesalpinia pluviosa also had the $95 \%$ ethanol extract of the dried bark active in the dose of $500 \mathrm{mg} / \mathrm{kg}$ to $P$. berghei infected mice and in vitro to $P$. falciparum - MRC $20\left(\mathrm{IC}_{50}=15 \mu \mathrm{g} /\right.$ $\mathrm{mL})$ and $P$. berghei $\left(\mathrm{IC}_{50}=8.3 \mu \mathrm{g} / \mathrm{mL}\right)$.

Species of plants of the Lauraceae family such Nectandra aff. hihua and Licaria canella had the 95\% ethanol extract active when evaluated against $P$. falciparum - MRC 20 and P. berghei in vitro and in vivo bioassays models. However, the $95 \%$ ethanol extract of the dried bark of Protium glabrescens (Burseraceae) in the $100 \mathrm{mg} / \mathrm{kg}$ dose was only active in vivo model to $P$. berguei, while it was inactive in vitro model to $P$. falciparum - MRC 20 and P. berghei (Deharo et al., 2001).

Also were related in scientific literature some species of the Solanaceae family with antimalarial activity. The plant Solanum nudum has been used by American community as Tumaco in Colombia to cure malaria and in according to Marcela et al. (2001), the aqueous extract obtained of the dried leaves and stem $(0.3$ $\% \mathrm{w} / \mathrm{v}$ - i.p.) was active against the $P$. berghei infected mice. The $95 \%$ ethanol extracts of the dried leaves of Dunalia brachyacantha and Saracha punctata were tested in vitro models for $P$. falciparum, so the first plant was active while the second one showed inactive. Both the extracts also were evaluated in vivo models to $P$. vinckei and all were active (Moretti et al., 1998; Munoz et al., 2000). However, the $95 \%$ ethanol and hexane extracts of Acnistus arborescens did not have this activity for $P$. berghei stain (Brandão et al., 1985).

Picrasma crenata (Veil.) Engl. (Simaroubaceae) is known as 'granadillo' or 'quasia', 'palo amargo' in Argentina and it is indicated such an antimalarial, febrifuge, tonic, antisyphilitic and insecticide. The aqueous and methanol extracts of the dried aerial parts this plant showed antimalarial activity in vitro for $P$. falciparum K-1 (Debenedetti et al., 2002). Other species from this botanical genus such $P$. excelsa and $P$. antillana did not have this activity for $P$. gallinaceum infected chicken when the methanol and chloroform extracts obtained from the wood were used (Spencer et al., 1947).

Other species used by American population did not have antimalarial activity in condition evaluated. Such the $80 \%$ ethanol extract of Strychnos pseudoquina (Loganiaceae) in the dose of $1,000 \mathrm{mg} / \mathrm{kg}$ (Andrade-Neto et al., 2003) and the aqueous extract obtained from the dried bark of Coutarea hexandra (Rubiaceae) when it was evaluated to $P$. berghei infected mice (Carvalho et al., 1991). In similar, Deianira erubescens (Gentianaceae) also did not show activity with the $80 \%$ ethanol extract obtained from the dried leaves and root (Andrade-Neto et al., 2003). The aqueous extract of the dried leaves of Lisianthus speciosus was active against $P$. berghei infected mice and in vitro for $P$. falciparum stain (Carvalho \& Krettli, 1991), while the same extract obtained of the dried root was inactive for $P$. berghei infected mice (Carvalho et al., 1991). In study elaborated by Diaz \& Medina (1996), the chloroform extract obtained from the dried stembark of Tabebuia ochracea ssp. neochrysantha (Bignoniaceae) which is traditionally used against malaria in the Amazon did not show bioactivity in vitro against $P$. berghei.

\section{CONCLUSION}

Plants represent a vital source to research new active principles for the treatment of human diseases such as malaria which is considered neglected and emergent in American countries because the high incidence of attacks of the female Anopheles mosquitoes infected with Plasmodium spp at risk of infection regions such the forests areas. In this paper a variety of plants of the American continent evaluated against the main protozoa causer of the malaria was related. However, we conclude that the plants with antimalarial activity are a few and they do not have this therapeutic property fully defined. Therefore, in this context, it is necessary to support financially the multidisciplinary and interdisciplinary research, mostly those related to natural products enabling the discovery of new antimalarial pharmaceuticals.

\section{ACKNOWLEDGMENTS}

The authors thanks to College of Pharmacy, the University of Illinois at Chicago, U.S.A., for helping with the computer database NAPRALERT. And are grateful to CNPq and CAPES for financial support and research fellowships.

\section{REFERENCES}

Agra MF, França PF, Barbosa-Filho JM 2007. Synopsis of the plants known as medicinal and poisonous in Northeast of Brazil. Rev Bras Farmacogn 17: 114-140.

Agra MF, Silva KN, Basílio IJLD, França PF, Barbosa-Filho JM 2008. Survey of medicinal plants used in the region Northeast of Brazil. Rev Bras Farmacogn 18: 472508.

Almeida RN, Navarro DS, Barbosa-Filho JM 2001. Plants with central analgesic activity. Phytomedicine 8: 310-322.

Alves JS, Castro JC, Freire MO, Cunha EVL, Barbosa-Filho JM, Silva MS 2000. Complete assignment of the $1 \mathrm{H}$ 
and 13C spectra of four triterpenes of the ursane, artane, lupane and friedelane groups. Magn Reson Chem 38: 201-206.

Amaral FMM, Ribeiro MNS, Barbosa-Filho JM, Reis AS, Nascimento FRF, Macedo RO 2006. Plants and chemical constituents with giardicidal activity. Rev Bras Farmacogn 16: 696-720.

Amorim CZ, Flores CA, Gomes BE, Marques AD, Cordeiro RSB 1988. Screening for antimalarial activity in the genus Pothomorphe. J Ethnopharmacol 24: 101-106.

Amorim CZ, Marques AD, Balão RS 1991. Screening of the antimalarial activity of plants of Cucurbitaceae family. Mem Inst Oswaldo Cruz 86: 177-180.

Andrade-Neto VF, Brandão MGL, Stehmann JR, Oliveira LA, Krettli AU 2003. Antimalarial activity of Cinchonalike plants used to treat fever and malaria in Brazil. $J$ Ethnopharmacol 87: 253-256.

Antoun MD, Gerena L, Milhous WK 1993. Screening of the flora of Puerto Rico for potential antimalarial bioactives. Int J Pharmacogn 31: 255-258.

Antoun MD, Ramos Z, Vazques J, Oquendo I, Proctor GR, Gerena L, Franzblau SG 2001. Evaluation of the flora of Puerto Rico for in vitro antiplasmodial and antimycobacterial activities. Phytother Res 15: 638624.

Banerjee JN, Lewis JJ 1954. Pharmacological studies in the Apocynaceous genus Aspidosperma Mart. \& Zucc., Aspidosperma oblongum A. D. C. J Pharm Pharmacol 6: 246-252.

Barbosa-Filho JM, Vasconcelos THC, Alencar AA, Batista LM, Oliveira RAG, Guedes DN, Falcão HS, Moura MD, Diniz MFFM, Modesto-Filho J 2005. Plants and their active constituents from South, Central, and North America with hypoglycemic activity. Rev Bras Farmacogn 15: 392-413.

Barbosa-Filho JM, Piuvezam MR, Moura MD, Silva MS, Lima KVB, Cunha EVL, Fechine IM, Takemura OS 2006a. Anti-inflammatory activity of alkaloids: a twenty century review. Rev Bras Farmacogn 16: 109-139.

Barbosa-Filho JM, Medeiros KCP, Diniz MFFM, Batista LM, Athayde-Filho PF, Silva MS, Cunha EVL, Almeida JRGS, Quintans-Júnior LJ 2006b. Natural products inhibitors of the enzyme acetylcholinesterase. Rev Bras Farmacogn 16: 258-285.

Barbosa-Filho JM, Martins VKM, Rabelo LA, Moura MD, Silva MS, Cunha EVL, Souza MFV, Almeida RN, Medeiros IA 2006c. Natural products inhibitors of the angiotensin converting enzyme (ACE). A review between 1980-2000. Rev Bras Farmacogn 16: 421446.

Barbosa-Filho JM, Nascimento-Júnior FA, Tomaz ACA, Athayde-Filho PF, Silva MS, Cunha EVL 2007a. Natural products with antileprotic activity. Rev Bras Farmacogn 17: 141-148.

Barbosa-Filho JM, Sena-Filho JG, Duringer JM, Uchoa DEA, Xavier HS, Braz-Filho R 2007b. Distribution of iridoid glucosides in plants from the genus Lippia (Verbenaceae): An investigation of Lippia alba (Mill.) N.E. Brown. Nat Prod Commun 2: 715-716.

Barbosa-Filho JM, Alencar AA, Nunes XP, Tomaz ACA, Sena-Filho JG, Athayde-Filho PF, Silva MS, Souza MFV, da-Cunha EVL 2008. Sources of alpha-, beta, gamma-, delta- and epsilon-carotenes: A twentieth century review. Rev Bras Farmacogn 18: 135-154.

Bastos JK, Gottlieb OR, Sarti SJ, Filho DS 1996. Isolation of lignans and sesquiterpenoids from leaves of Zanthoxylum naranjillo. Nat Prod Lett 9: 65-70.

Becker ER 1949. Report on the thirty-five drugs and three plant materials tested against Plasmodium lophurae in the white pekin duck. Iowa State Coll J Sci 23: 189.

Blair S, Mesa J, Correa A, Carmona-Fonseca J, Granados H, Saez J 2002. Antimalarial activity of neurolenin B and derivatives of Eupatorium inulaefolium (Asteraceae). Pharmazie 57: 413-415.

Brandão M, Botelho M, Krettli E 1985. Antimalarial experimental chemotherapy using natural products. Cienc Cult 37: 1152-1163.

Brandão MGL, Grandi TSM, Rocha EMM, Sawyer DR, Krettli AU 1992. Survey of medicinal plants used as antimalarials in the Amazon. $J$ Ethnopharmacol 36: 175-182.

Brandão MGL, Krettli AU, Soares LSR, Nerey CGC, Marinuzzi HC 1997. Antimalarial activity of extracts and fractions from Bidens pilosa and other Bidens species (Asteraceae) correlated with the presence of acetylene and flavonoid compound. J Ethnopharmacol 57: 131138.

Bravo JA, Sauvain M, Gimenez A, Munoz V, Callapa J, Le Men-Olivier L, Massiot G, Lavaud C 1999. Bioactive phenolic glycosides from Amburana cearensis. Phytochemistry 50: 71-74.

Bray DH, Warhurst DC, Connolly JD, O’Neill MJ, Phillipson JD 1990. Plants as sources of antimalarial drugs. Part 7. Activity of some species of Meliaceae plants and their constituent limonoids. Phytother Res 4: 29-35.

Bustillos F, Flores Y, Almanza G 2002. Triterpenoids from Polypodium decumanum. Rev Boliv Quim 19: 34-38.

Cabral JA, Mc Chesney JD, Milhous WK 1993. A new antimalarial quassinoid from Simaba guianensis. $J$ Nat Prod 56: 1954-1961.

Calderon AI, Angerhofer CK, Pezzuto JM, Farnsworth NR, Foster R, Condit R, Gupta MP, Soejarto DD 2000. Forest plot as a tool to demonstrate the pharmaceutical potential of plants in a tropical forest of Panama. Econ Bot 54: 278-294.

Camacho MR, Phillipson JD, Croft SL, Marley D, Kirby G, Dwarhurs DC 2002. Assessment of the antiprotozoal activity of Galphimia glauca and the isolation of new nor-secofriedelanes and norfriedelanes. J Nat Prod 65: 1457-1461.

Carvalho LH, Brandão MGL, Santos-Filho D, Lopes JLC, Krettli AU 1991. Antimalarial activity of crude extracts from brazilian plants studies in vivo in Plasmodium berghei-infected mice and in vitro against Plasmodium falciparum in culture. Braz J Med Biol Res 24: 1113-1123.

Carvalho LH, Krettli AU 1991. Antimalarial chemotherapy with natural products and chemically defined molecules. Mem Inst Oswaldo Cruz 86: 181-184.

Carvalho LH, Ferrari WMS, Krettli AU 1992. A method for screening drugs against the liver stages of malaria using Plasmodium gallinaceum and Aedes mosquitos. Braz J Med Biol Res 25: 247-255.

Chaturvedula VSP, Farooq A, Schilling JK, Malone S, Derveld I, Werkhoven MCM, Wisse JH, Ratsimbason M, Kingston DGI 2004. New eudesmane derivatives from Melampodium camphoratum from the Suriname rainforest. J Nat Prod 67: 2053-2057.

De Almeida Alves TM, Nagem TJ, De Carvalho CL, Krettli AU, Zani CL 1997. Antiplasmodial triterpene from Vernonia brasiliana. Planta Med 63: 554-555.

Debenedetti S, Muschietti LM, Baren CV, Clavin M, Broussalis A, Martino V, Houghton PJ, Warhurst D, Steele J 2002. In vitro antiplasmodial activity of extracts of Argentinian plants. J Ethnopharmacol 80: 163-166.

Deharo E, Bourdy G, Quenevo C, Munoz V, Ruiz G, Sauvain 
M 2001. A search for natural bioactive compounds in Bolivia through a multidisciplinary approach. Part V. Evaluation of the antimalarial activity of plants used by the Tacana Indians. $J$ Ethnopharmacol 77: 91-98.

Diaz F, Medina JD 1996. Furanonaphthoquinones from Tabebuia ochracea ssp. neochrysanta. J Nat Prod 59: 423-424.

Dominguez JA 1932. Malaria treatment with Aspidosperma Quebracho Blanco. Rev Farm (Buenos Aires) 73: 82.

Falcão HS, Lima IO, Santos VL, Dantas HF, Diniz MFFM, Barbosa-Filho JM, Batista LM 2005. Review of the plants with anti-inflammatory activity studied in Brazil. Rev Bras Farmacogn 15: 381-391.

Falcão HS, Mariath IR, Diniz MFFM, Batista LM, BarbosaFilho JM 2008a. Plants of the American continent with antiulcer activity. Phytomedicine 15: 132-146.

Falcão HS, Leite JA, Barbosa-Filho JM, Athayde-Filho PF, Chaves MCO, Moura MD, Ferreira AL, Almeida ABA, Souza-Brito ARM, Diniz MFFM, Batista LM 2008b. Gastric and duodenal antiulcer activity of alkaloids: a review. Molecules 13: 3198-3223.

Federici E, Palazzino G, Nicoletti M, Galeffi C 2000. Antiplasmodial activity of the alkaloids of Peschiera fuchsiaefolia. Planta Med 66: 93-95.

Francois G, Passreiter CM, Woerdenbag HJ, Van Looveren M 1996. Antiplasmodial activities and cytotoxic effects of aqueous extracts and sesquiterpene lactones from Neurolaena lobata. Planta Med 62: 126-129.

Franssen FFJ, Simeijsters LJJW, Berger I, Aladan BEM 1997. In vivo and vitro antiplasmodial activities of some plants traditionally used in Guatemala against malaria. Antimicrob Agents Chemother 41: 1500-1503.

Froelich S, Onegi B, Kakooko A, Siems K, Schubert C, JenettSiems K 2007. Plants traditionally used against malaria: phytochemical and pharmacological investigation of Momordica foetida. Rev Bras Farmacogn 17: 1-7.

Froelich S, Gupta MP, Siems K, Jenett-Siems K 2008. Phenylethanoid glycosides from Stachytarpheta cayennensis (Rich.) Vahl, Verbenaceae, a traditional antimalarial medicinal plant. Rev Bras Farmacogn 18: 517-520.

Gantier JC, Fournet A, Munos MH, Hocquemiller R 1996. The effect of some 2-substituted quinolines isolated from Galipea longiflora on Plasmodium vinckei Petteri infected mice. Planta Med 62: 285-286.

Gertsch J, Niomawe, Gertsch-Roost K, Sticher O 2004. Phyllanthus piscatorum, ethnopharmacological studies on a women's medicinal plant of the Yanomami Amerindians. J Ethnopharmacol 91: 181-188.

Gonçalves MCR, Moura LSA, Rabelo LA, Barbosa-Filho JM, Cruz HMM, Cruz J 2000. Natural products inhibitors of HMG CoA reductase. Rev Bras Farm 81: 63-71.

Gonçalves MCR, Melo Diniz MFFM, Borba JDC, Nunes XP, Barbosa-Filho JM 2006. Berinjela (Solanum melongena L.) - mito ou realidade no combate as dislipidemias? Rev Bras Farmacogn 16: 252-257.

Harrison TR, Fauci AS, Braunwald E, Isselbacher KJ, Wilson JD, Martin JB, Kasper DL, Hauser SL, Longo DL 1998. Medicina Interna. 14.ed. Rio de Janeiro: Mc Graw Hill.

Hernandez H, Mendiola J, Torress D, Garrido N, Perez N 1990. Effect of aqueous extracts of Artemisia on the in vitro culture of Plasmodium falciparum. Fitoterapia 61: 540-541.

Jacquemond-Collet I, Benoit-Vical F, Alexix Valenti M, Stanislas E, Mallie M, Fouraste I 2002. Antiplasmodial and cytotoxic activity of galipine and other tetrahydroquinolines from Galipea officinalis. Planta
Med 68: 68-69.

Jenett Siems K, Siems K, Jakupovic J, Solis PN, Gupta MP, Mockenhaupt FP, Bienzle U, Eich E 2000. Sipandinolide: a butenolide including a novel type of carbon skeleton from Siparuna andina. Planta Med 66: 384-385.

Jensen JF, Kvist LP, Christensen SB 2002. An antiplasmodial lignan from Euterpe precatoria. J Nat Prod 65: 19151917.

Kohler I, Lenett Siems K, Mockenhaupt FP, Siems K, Jakupovic J, Gonzalez JC, Hernandez MA, Ibarra RA, Berensohn WG, Bienzle U, Eich E 2001. In vitro antiplasmodial activity of 4-phenylcoumarins from Exostema mexicanum. Planta Med 67: 89-91.

Kraft C, Jenett Siems K, Siems K, Gupta MP, Bienzle U, Eich E 2000. Antiplasmodial activity of isoflavones from Andira inermis. J Ethnopharmacol 73: 131-135.

Kraft C, Jenett Siems K, Siems K, Solis PN, Gupta MP, Bienzle U, Eich E 2001. Andinermals A-C, antiplasmodial constituents from Andira inermis. Phytochemistry 58 : 769-774.

Krettli AU, Andrade-Neto VF, Brandão MDGL, Ferrari WMS 2001a. The search for new antimalarial drugs from plants used to treat fever and malaria or plants ramdomly selected: a review. Mem Inst Oswaldo Cruz 96: 1033-1042.

Krettli AU, Brandão MDGL, Ferrari WMS 2001b. New antimalarial drugs: a search based on plants used in popular medicine to treat fever and malaria. Folha Med 120: 119-126.

Levander OA, Ager AL, Morris VC, May RG 1991. Protective effect of ground flaxseed or ethyl linolenate in a vitamin e-deficient diet against murine malaria. Nutr Res 11: 941-948.

Loizaga NS, Sagastume LC 1935. Malaria treatment with "Quechuol-Dominguez". Semana Med Buenos Aires 562-566.

Lopes NP, Kato MJ, Andrade EHDEA, Maia JS, Yoshida M, Planchart AR, Katzin AM 1999. Antimalarial use of volatile oil from leaves of Virola surinamensis (Rol.) Warb. by Waiapi Amazon Indians. J Ethnopharmacol 67: 313-319.

Lovy A, Knowles B, Labbe R, Nolan L 1999. Activity of Edible mushrooms against the growth of human T4 leukemic cancer cells, HELA cervical cancer cells and Plasmodium falciparum. J Herbs Spices Med Plants 6: 49-57.

MacKinnon S, Durst T, Arnason JT, Angerhofer CJ, Pezzuto JM, Sanchez-Vindas PE, Poveda LJ, Gbeassor M 1997. Antimalarial activity of tropical Meliaceae extracts and Gedunin derivatives. J Nat Prod 60: 336-341.

Makler MT 1994. The effect of cocaine on the growth of Plasmodium falciparum in vitro. Trans Roy Soc Trop Med Hyg 88: 444.

Malagon F, Vazquez J, Dalgado G, Ruiz A 1997. Antimalaric effect of an alcoholic extract of Artemisia ludoviciana Mexicana in a rodent malaria model. Parasitology 39: 3-7.

Marcela E, Silvia B, Jaime C, Pilar P 2001. Effect of Solanun nudum extracts on the liver of mice infected with Plasmodium berghei. Amer J Chinese Med 29: 477484.

Mcphail KL, Correa J, Linington RG, Gonzalez J, Ortega-Barria E, Capson TL, Gerwick WH 2007. Antimalarial linear lipopeptides from a Panamanian strain of the marine Cyanobacterium Lyngbya majuscula. J Nat Prod 70: 984-988.

Mess J, Blair S, Saez J, Correa A, Carmona J 1998. In 
vitro antimalarial evaluation of extracts of plants Alternanthera. An Quim Int Ed 92: 67-70.

Montenegro LHM, Oliveira PES, Conserva LM, Rocha EMM, Brito AC, Araujo RM, Trevisan MTS, Lemos RPL 2006. Terpenoids and evaluation of the antimalarial, larvicidal, anti-radicalar and anticholinesterase potential of Pouteria venosa (Sapotaceae). Ver Bras Farmacogn 16: 611-617.

Morais LCSL, Barbosa-Filho JM, Almeida RN 2003. Plants and bioactive compounds for the treatment of Parkinson's disease. Arquivos Brasileiros de Fitomedicina Cientifica 1: 127-132.

Moretti C, Sauvain M, Lavaud C, Massiot G, Bravo JA, Munoz $\mathrm{V}$ 1998. A novel antiprotozoal aminosteroid from Saracha punctata. J Nat Prod 61: 1390-1393.

Mota KSL, Dias GEN, Pinto MEF, Luiz-Ferreira A, SouzaBrito ARM, Hiruma-Lima CA, Barbosa-Filho JM, Batista LM 2009. Flavonoids with gastroprotective activity. Molecules 14: In Press, DOI: 10.3390/ molecules 140x000x.

Moura MD, Torres AR, Oliveira RAG, Diniz MFFM, BarbosaFilho JM 2001. Natural products as inhibitors of models of mammary neoplasia. Brit J Phytother 5: 124-145.

Moura MD, Silva JS, Oliveira RAG, Diniz MFFM, BarbosaFilho JM 2002. Natural products reported as potential inhibitors of uterine cervical neoplasia. Acta Farm Bonaerense 21: 67-74.

Muhammad I, Dunbar DC, Takamatsu S, Walker LA, Clark AM 2001. Antimalarial, cytotoxic, and antifungal alkaloids from Duguetia hadrantha. J Nat Prod 64: 559-562.

Muhammad I, Dunbar DC, Khan SI, Tekwani BL, Bedir E, Takamatsu S, Ferreira D, Walker LA 2003. Antiparasitic alkaloids from Psychotria klugii. J Nat Prod 66: 962-967.

Munoz V, Sauvain M, Bourdy G, Arrazola S, Callapa J, Ruiz G, Choque J, Deharo E 2000. A search for natural bioactive compounds in Bolivia through a multidisciplinary approach part III. Evaluation of the antimalarial activity of plants used by Altenos Indians. J Ethnopharmacol 71: 123-131.

Neves DP, Melo AL, Genaro O, Linardi PM 2005. Parasitologia Humana. 11.ed. Rio de Janeiro: Atheneu.

Noster S, Kraus LJ 1990. In vitro antimalarial activity of Coutarea latiflora and Exostema caribaeum extracts on Plasmodium falciparum. Planta Med 56: 63-65.

Okunade AL, Bikoff RE, Casper SJ, Oksman A, Goldberg DE, Lewis WH 2003. Antiplasmodial activity of extracts and quassinoids isolated from seedlings of Ailanthus altissima (Simaroubaceae). Phytother Res 17: 675677.

Okunade AL, Lewis WH 2004. Oleanene constituents of Lantana cujabensis. Fitoterapia 75: 327-331.

Oliveira FQ, Andrade-Neto V, Krettli AU, Brandão MGL 2004. New evidences of antimalarial activity of Bidens pilosa root correlated with polyacetylene and flavonoids. J Ethnopharmacol 93: 39-42.

O’Neill MJ, Bray DH, Boardman P, Phillipson JD, Warhurst DC 1985. Plants as sources of antimalarial drugs. Part.1. In vitro test method for the evaluation of crude extracts from plants. Planta Med 51: 394-398.

O’Neill MJ, Bray DH, Boardman P, Wright CW, Phillipson JD, Warhurst DC, Gupta MP, Correya M, Solis P 1987. The activity of Simarouba amara against chloroquineresistant Plasmodium falciparum in vitro. J Pharm Pharmacol Suppl 39: 80P

O’Neill MJ, Bray DH, Boadman P, Wright CW, Phillipson JD, Warhurst DC, Gupta MP, Correya M, Solis P 1988.
Plants as sources of antimalarial drugs. Part 6: activities of Simarouba amara fruits. J Ethnopharmacol 22: 183-190.

OPS 1997. Organizatión Panamericana de la Salud. Indicadores Básicos. Situación de Salud en las Américas.

Palmeira Júnior SF, Conserva LM, Barbosa-Filho JM 2006. Clerodane diterpenes from Croton species: Distribution and the compilation of their ${ }^{13} \mathrm{C} \mathrm{NMR}$ spectral data. Nat Prod Commun 1: 319-344.

Pereira JV, Modesto-Filho J, Agra MF, Barbosa-Filho JM 2002. Plant and plant-derived compounds employed in prevention of the osteoporosis. Acta Farm Bonaerense 21: 223-234.

Phillipson JD, O’Neill MJ 1986. Novel antimalarial drugs from plants? Parasitology 2: 355-358.

Quintans-Júnior LJ, Almeida JRGS, Lima JT, Nunes XP, Siqueira JS, Oliveira LEG, Almeida RN, Athayde-Filho PF, Barbosa-Filho JM 2008. Plants with anticonvulsant properties - a review. Rev Bras Farmacogn 18 (Supl.): 798-819.

Rates SMK 2001. Plants as source of drugs. Toxicon 39: 603613.

Rocha LG, Almeida JRGS, Macedo RO, Barbosa-Filho JM 2005. A review of natural products with antileishmanial activity. Phytomedicine 12: 514-535.

Seeler AO, Dusenbery E, Malanga C 1943. The comparative activity of quinine, quinidine, cinchonine, cinchonidine and quinoidine against Plasmodium lophurae infections of pekin ducklings. J Pharmacol Exp Ther 78: 159-163.

Sena-Filho JG, Duringer JM, Maia GLA, Tavares JF, Xavier HS, Silva MS, Cunha EVL, Barbosa-Filho JM 2008. Ecdysteroids from Vitex species: Distribution and compilation of their 13C-NMR spectral data. Chem Biodivers 5: 707-713.

Silva JS, Moura MD, Oliveira RAG, Diniz MFFM, BarbosaFilho JM 2003. Natural product inhibitors of ovarian neoplasia. Phytomedicine 10: 221-232.

Sousa FCF, Melo CTV, Citó MCO, Félix FHC, Vasconcelos SMM, Fonteles MMF, Barbosa-Filho JM, Viana GSB 2008. Plantas medicinais e seus constituintes bioativos: Uma revisão da bioatividade e potenciais benefícios nos distúrbios da ansiedade em modelos animais. Rev Bras Farmacogn 18: 642-654.

Souza EL, Stamford TLM, Lima EO, Trajano VN, Barbosa Filho JM 2005. Antimicrobial effectiveness of spices: an approach for use in food conservation systems. Braz Arch Biol Technol 48: 549-558.

Spencer CF, Koniuszy FR, Rogers EF, Shavel Junior J, Easton NR, Kaczka EA, Kuehl Junior FA, Phillips RF, Walti A, Folkers K, Malanga C, Seeler AO 1947. Survey of plants for antimalarial activity. Lloydia 10: 145-174.

Steele JCP, Veitch NC, Kite GC, Simmonds MSJ, Warhurst DC 2002. Indole and beta-carboline alkaloids from Geissospermum sericeum. J Nat Prod 65: 85-88.

Trigger PI, Kondrachine AV 1998. Commentary: Malaria control in the 1990s. Bull WHO 76: 11.

Ueno HM, Doyama JT, Padovani E, Salata CR 1996. Effect of Momordica charantia L. in mice infected with Plasmodium berghei. Rev Soc Bras Med Trop 29: 455-460.

Veronesi R. 1991. Doenças Infecciosas e Parasitárias. 8.ed. Rio de Janeiro: Guanabara Koogan.

Wasicky R, Unti O, Barbieri E 1942. Quinine and alkaloids in Brazil. An Fac Farm Odontol Univ Sao Paulo 3: 137.

WHO 1997. World Health Organization. World malaria situation in 1994. Wkly Epidemiol Rec 1992; 67: 161-167. 


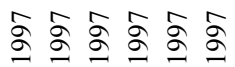

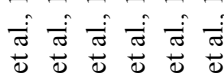

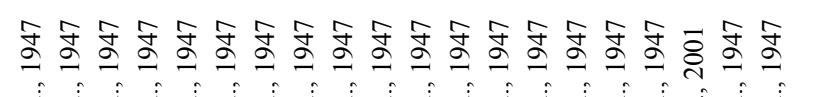

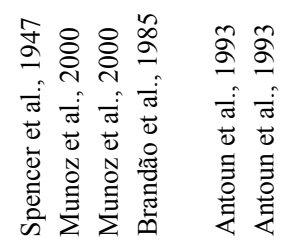

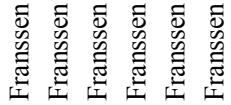

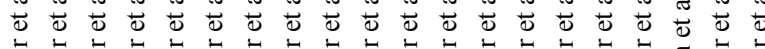

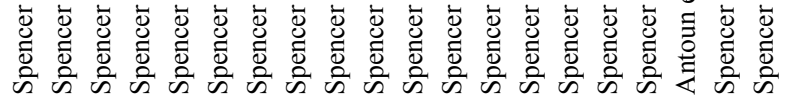

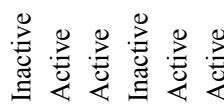

总

总总总

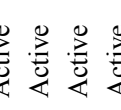

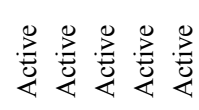

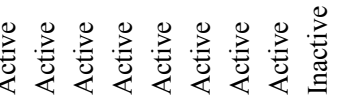

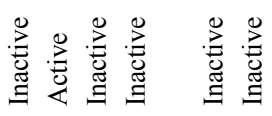
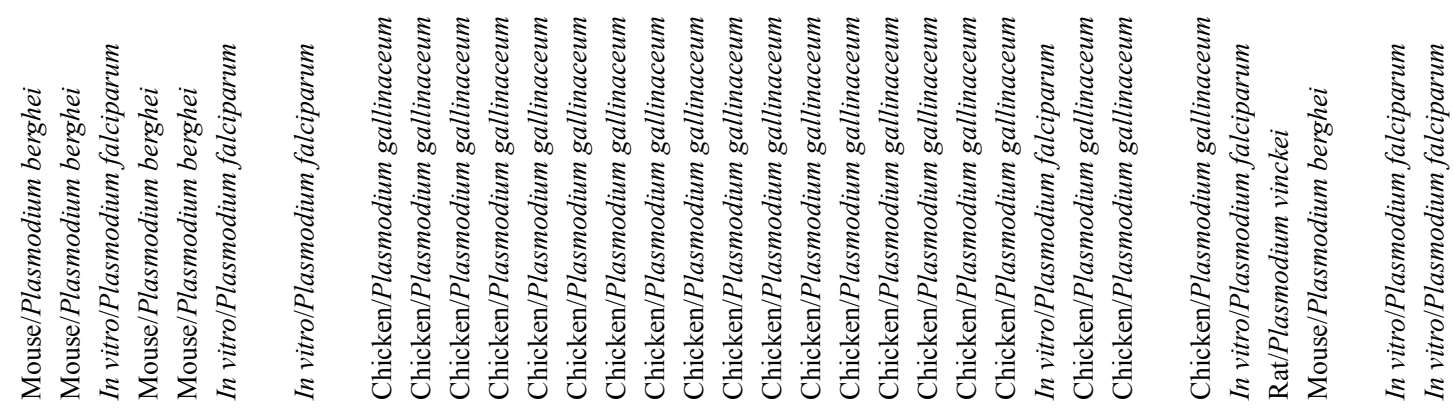

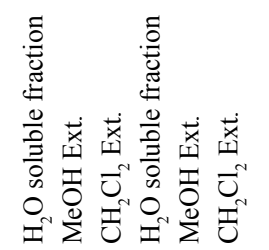

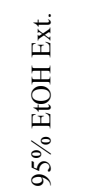

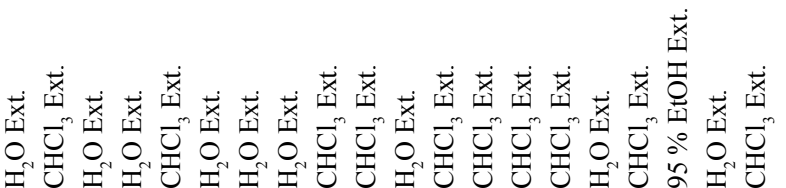

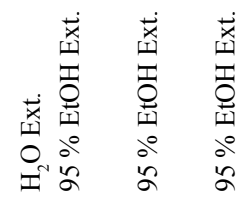

突

惫

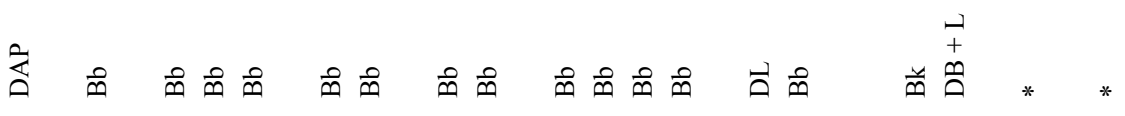

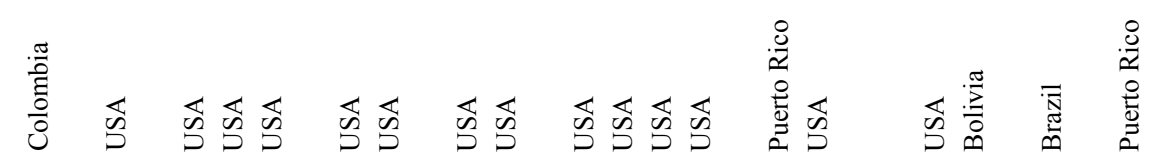

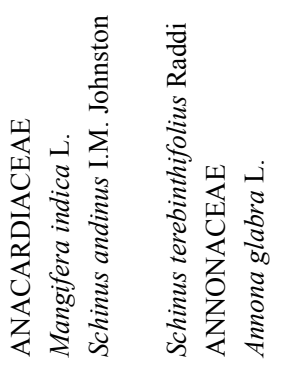



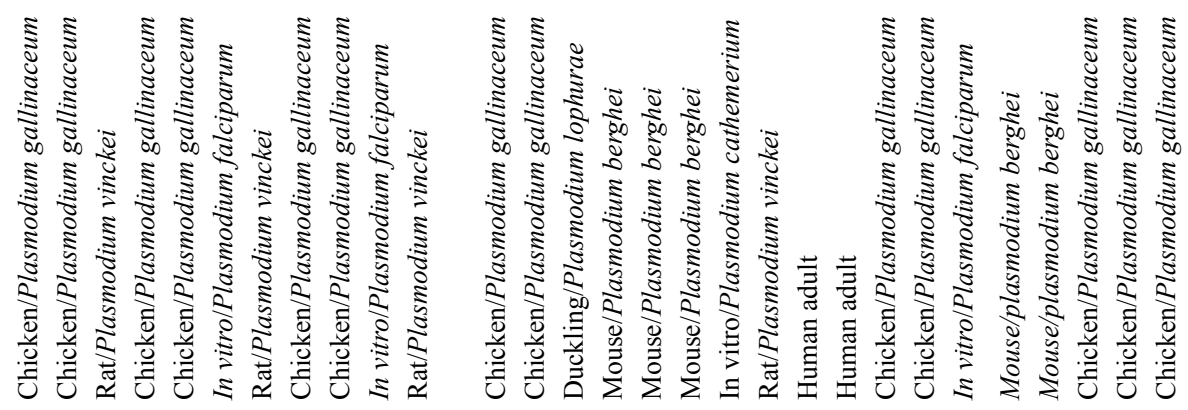

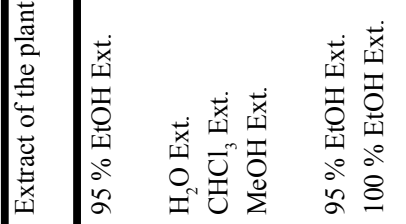
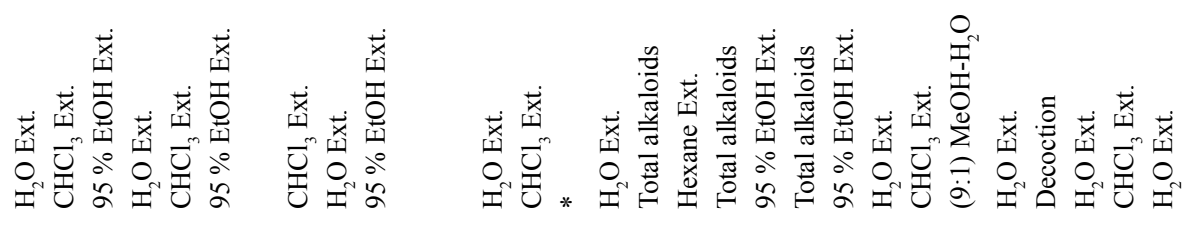

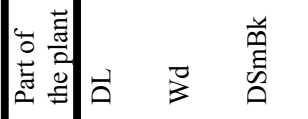

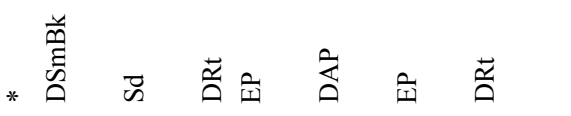

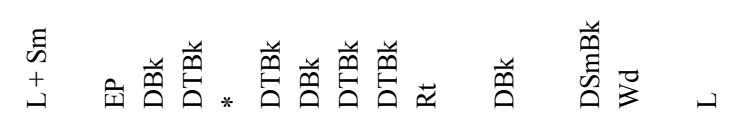

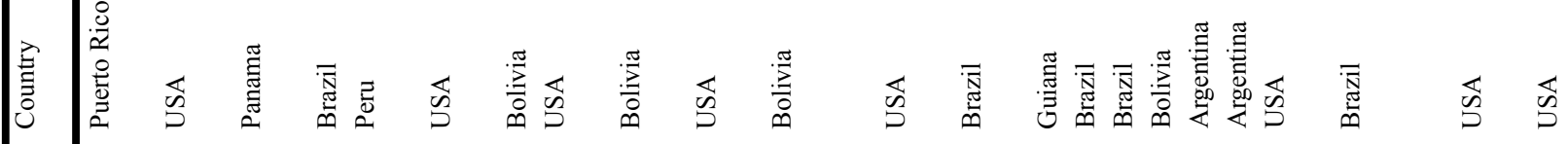
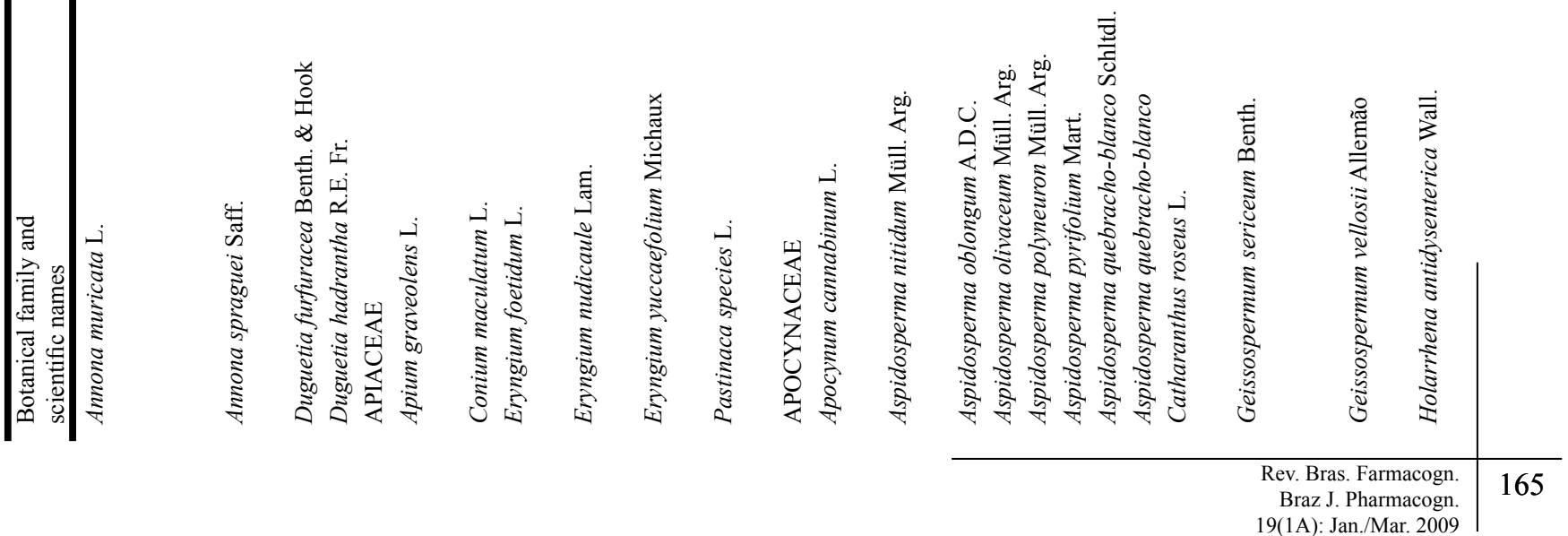


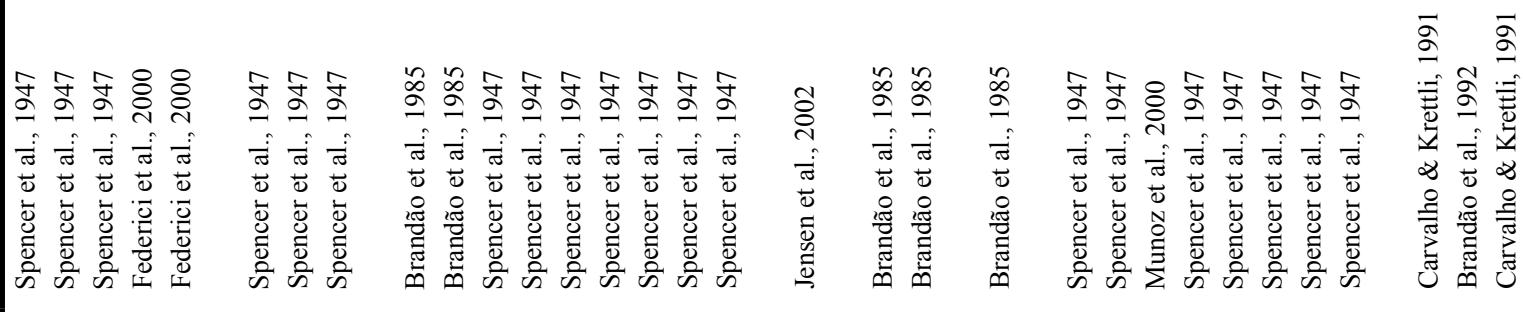

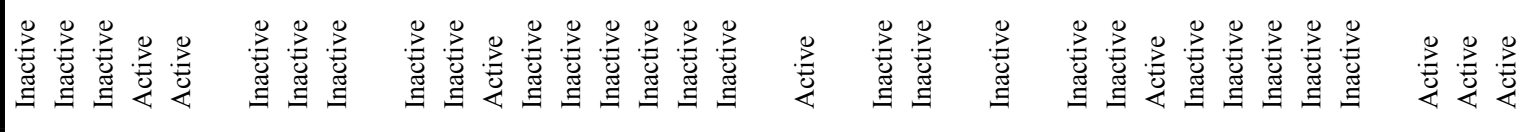
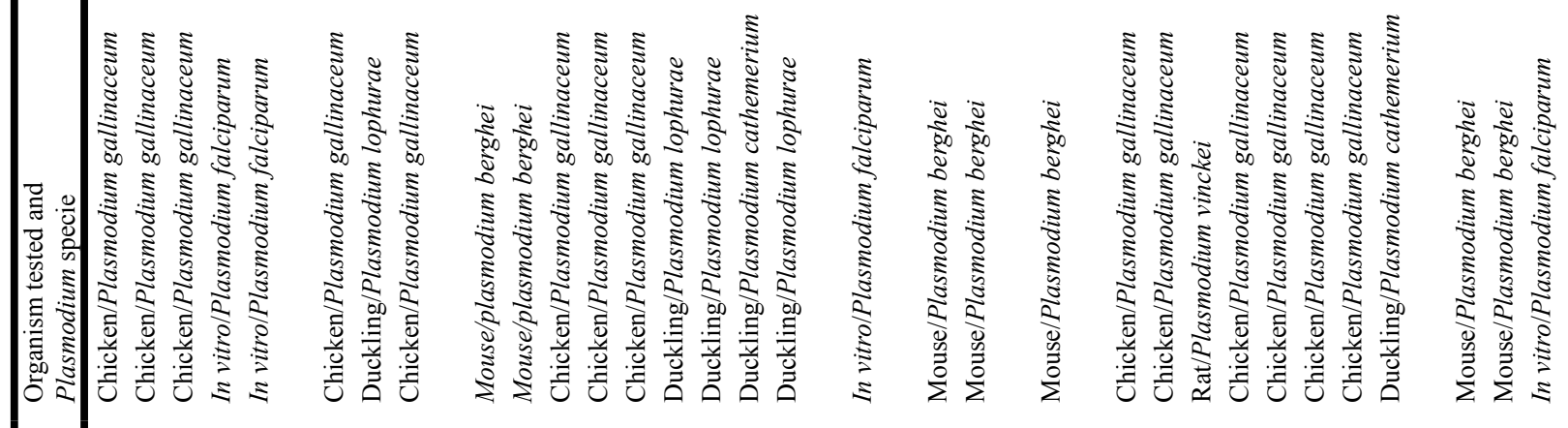

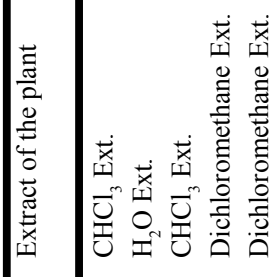

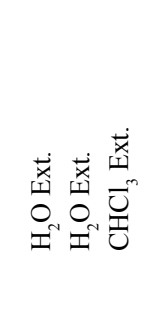

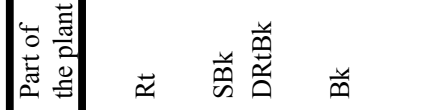

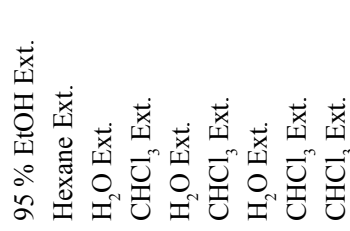

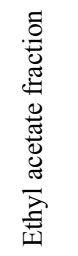

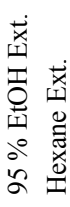

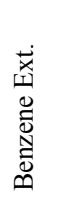

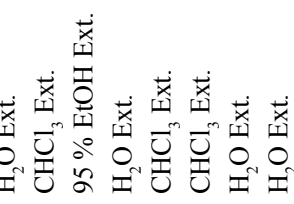

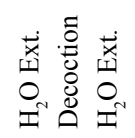

* $\quad \begin{gathered}\text { ह } \\ + \\ +\end{gathered}$

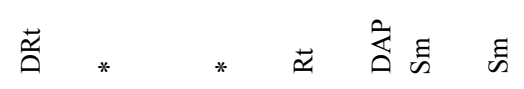

望

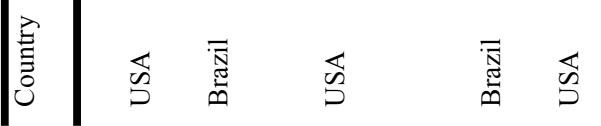

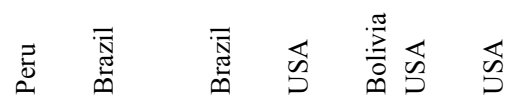

:

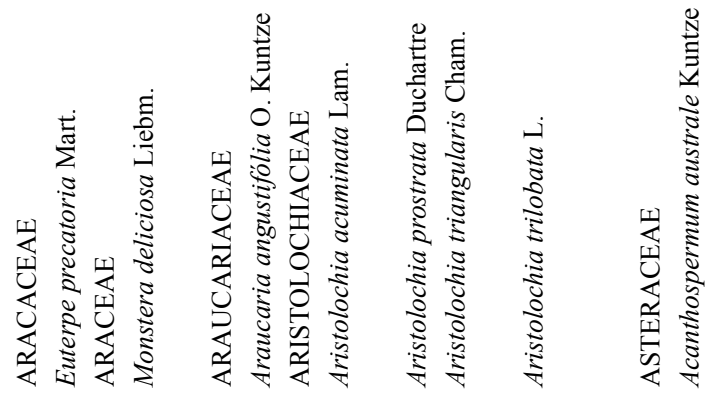




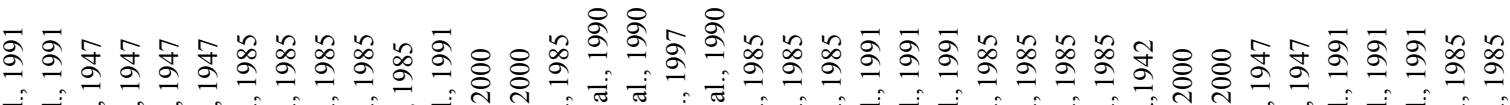

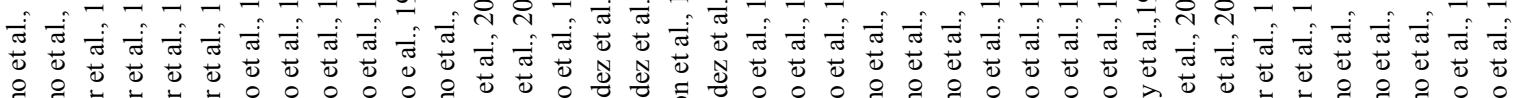

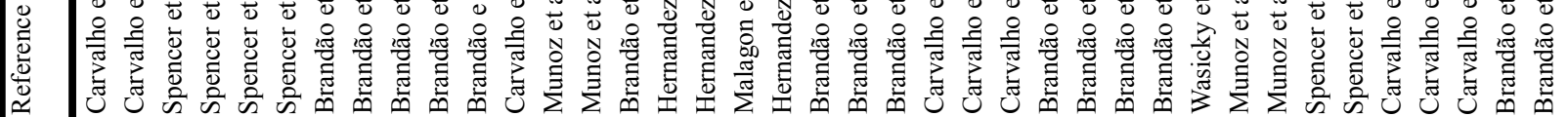

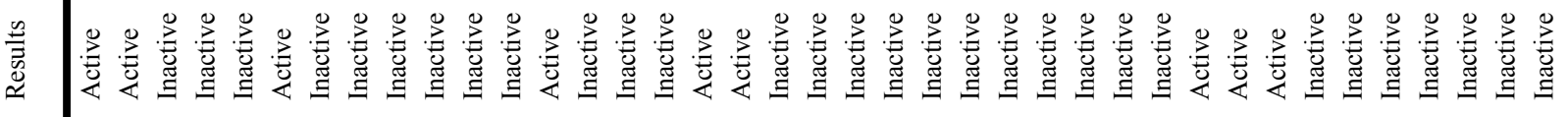

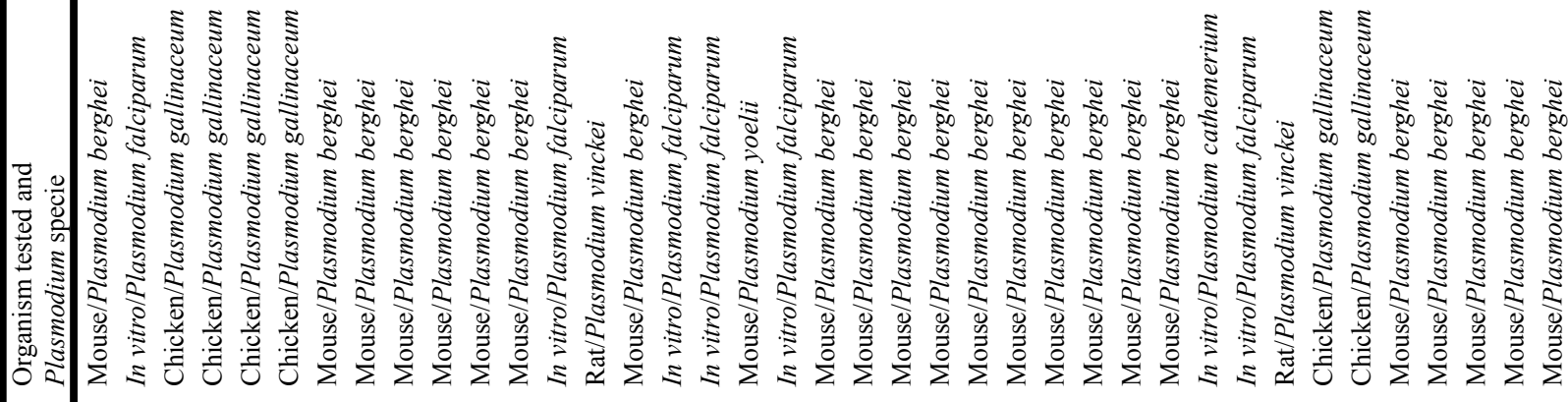

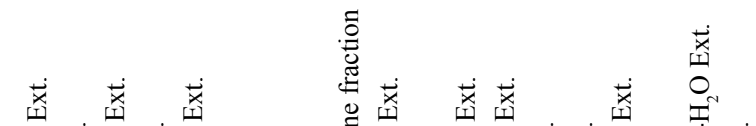

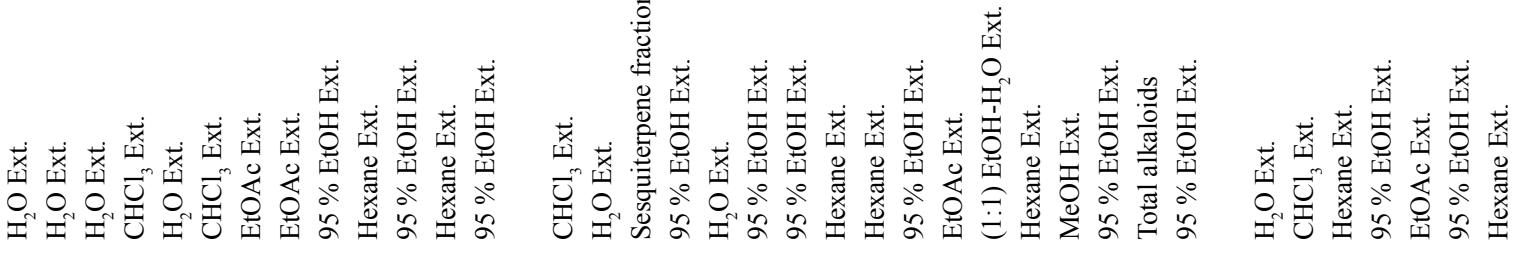
泀

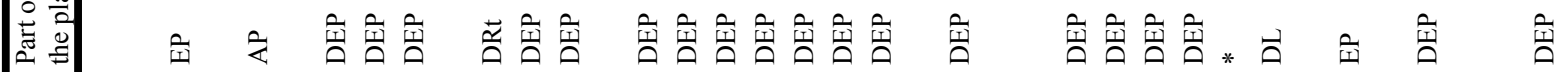

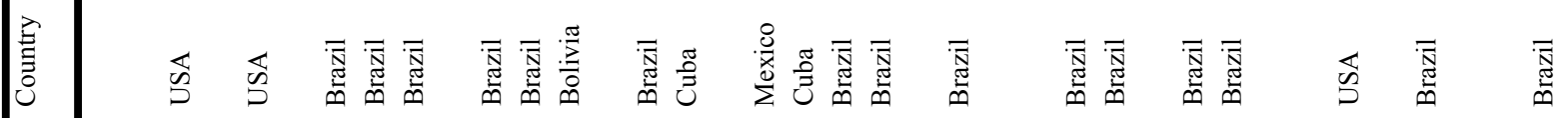




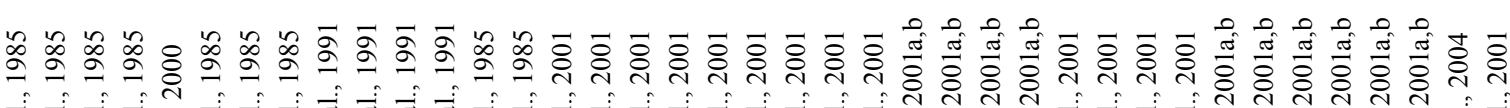

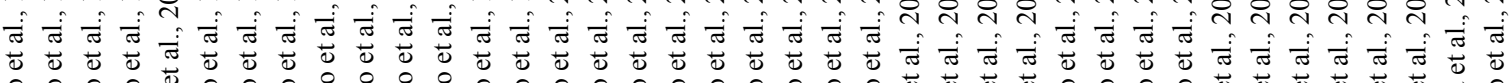

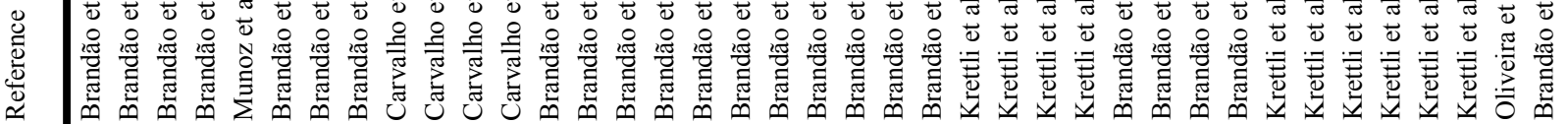

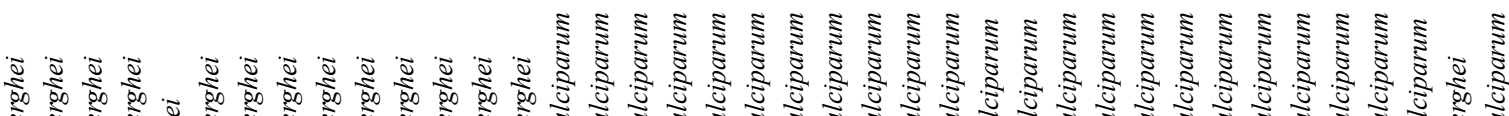

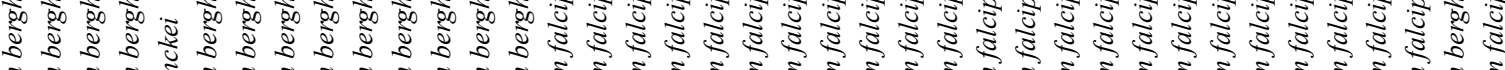

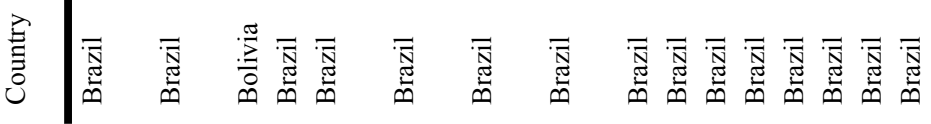




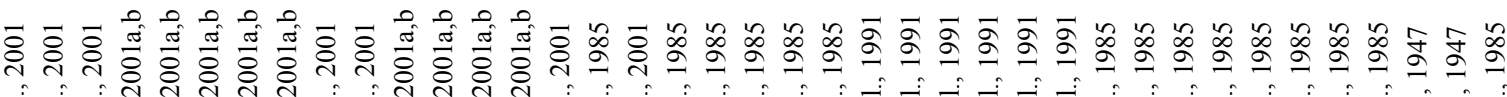

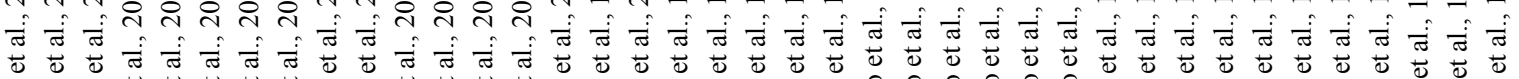

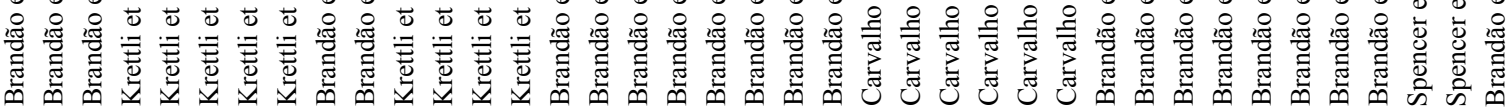




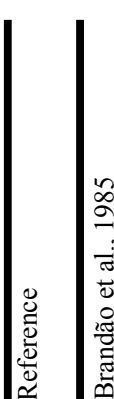

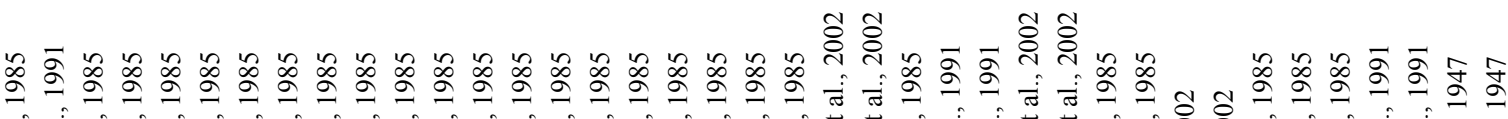

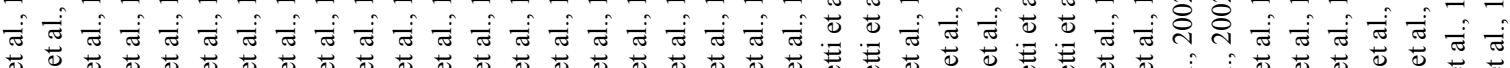

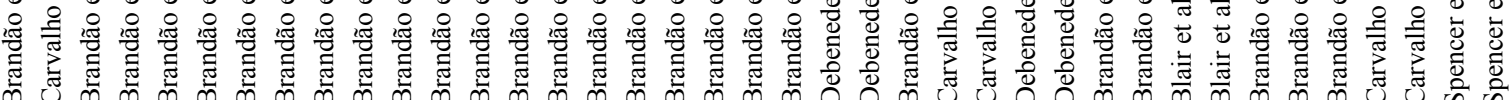

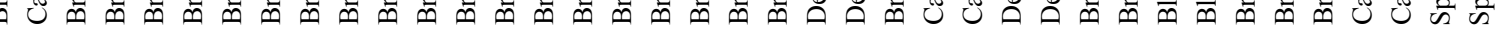

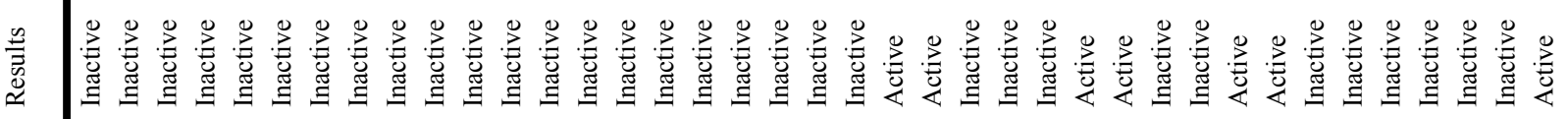

蒙

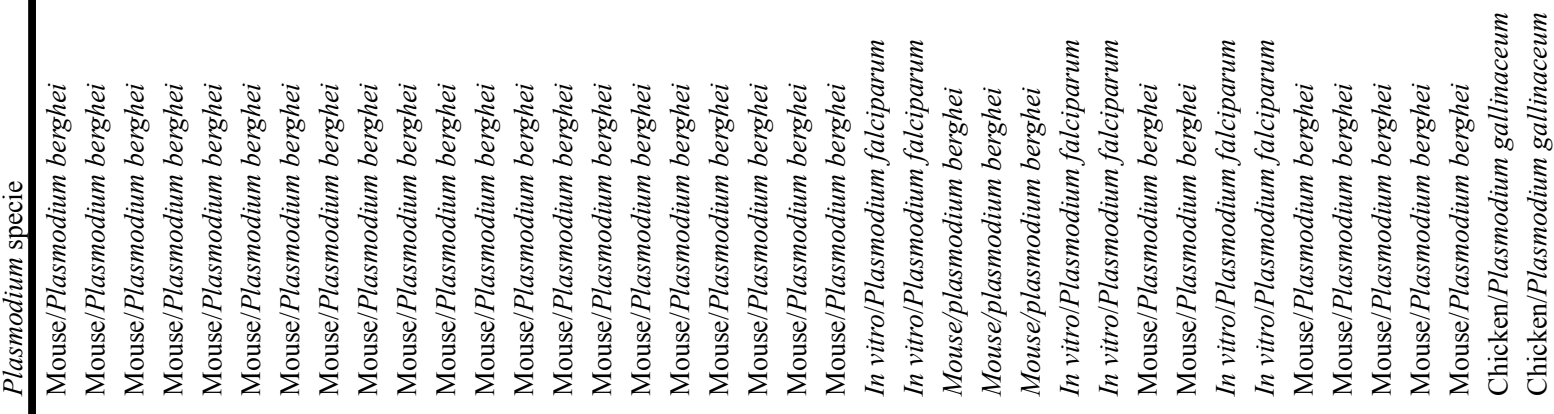

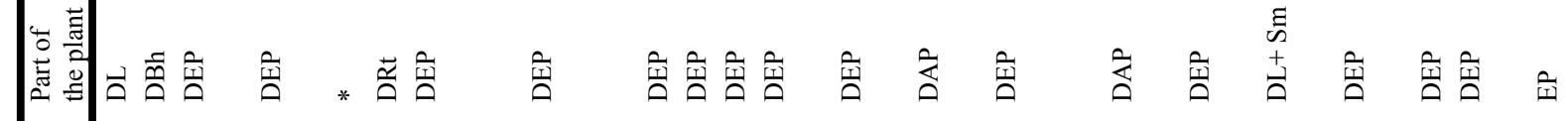

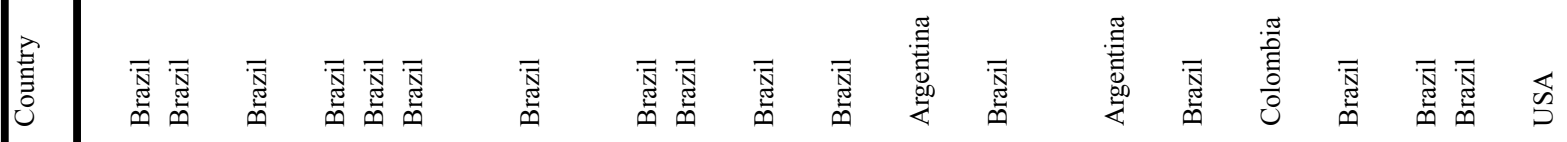




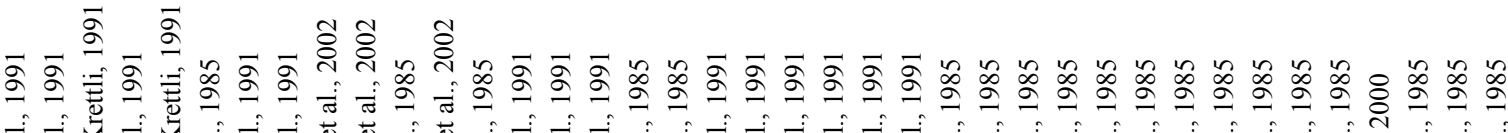

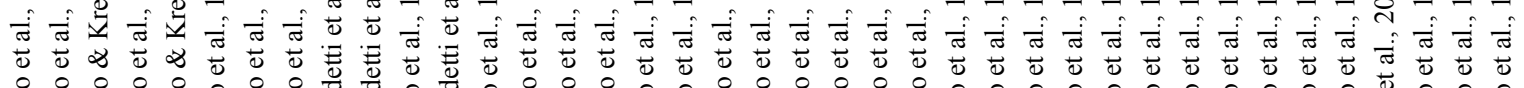

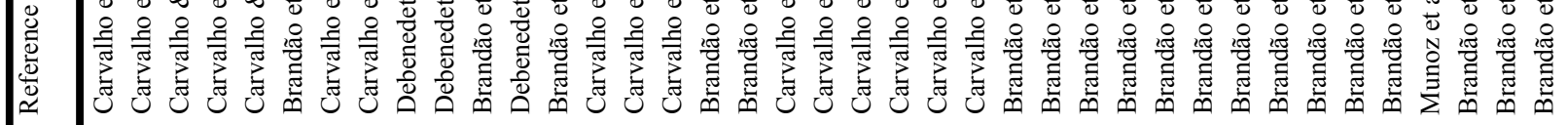


$\stackrel{4}{\stackrel{4}{*}}$

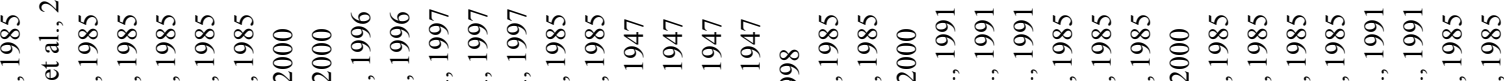

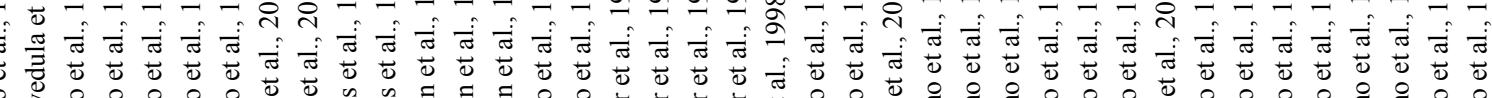

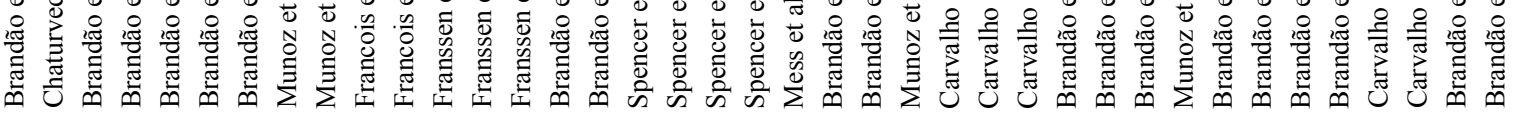

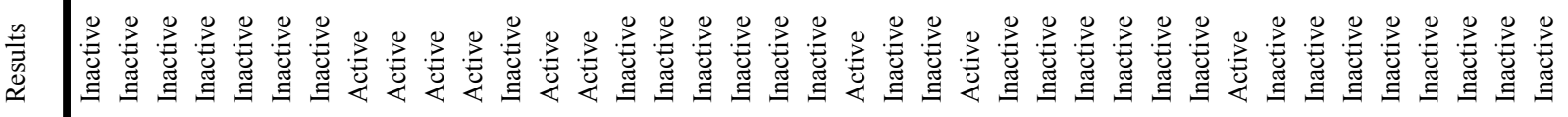

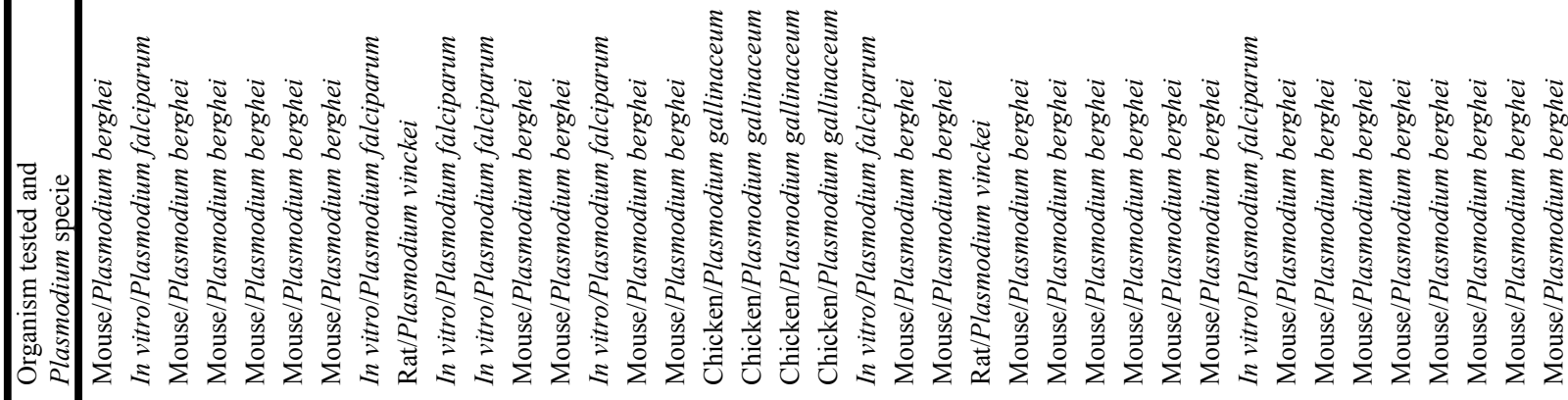

䒬

西

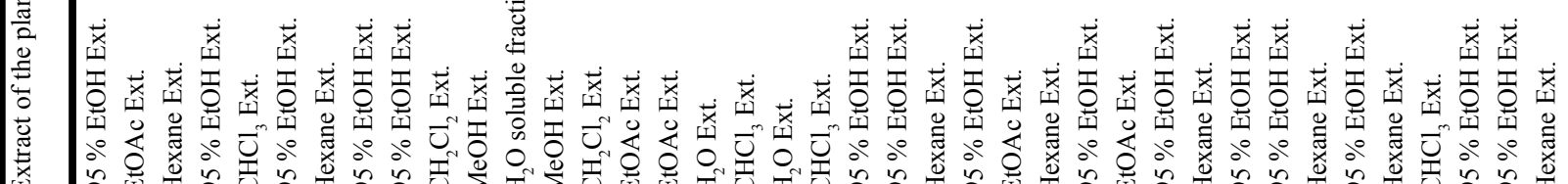

- 泀

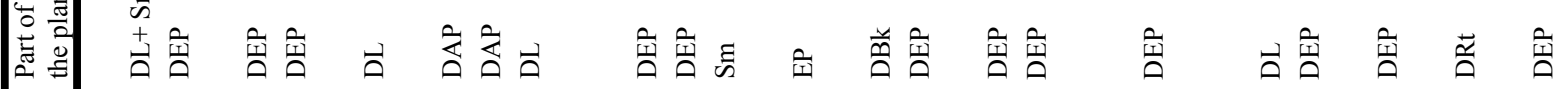

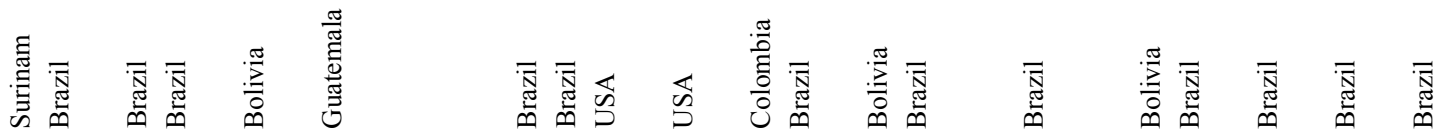

\begin{tabular}{|c|c|c|c|c|c|c|c|}
\hline 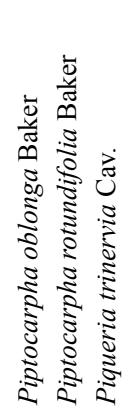 & 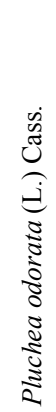 & 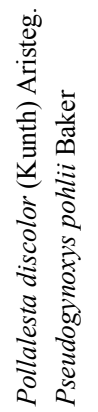 & 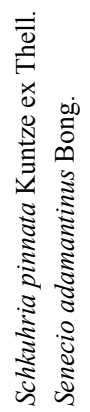 & 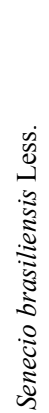 & 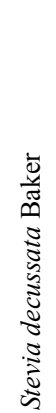 & 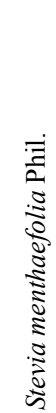 & 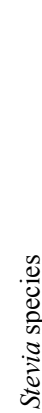 \\
\hline
\end{tabular}




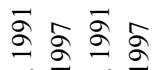

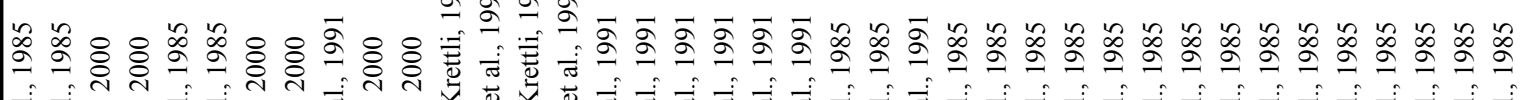

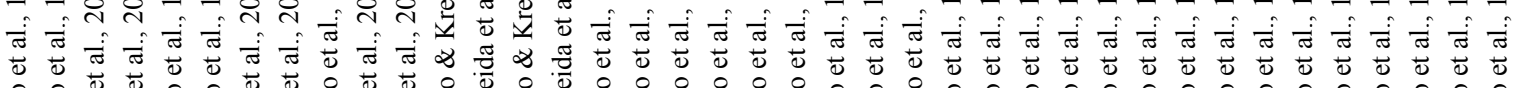

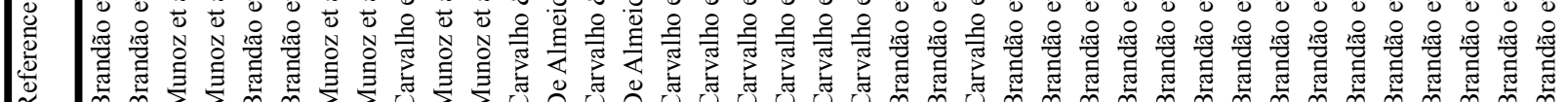

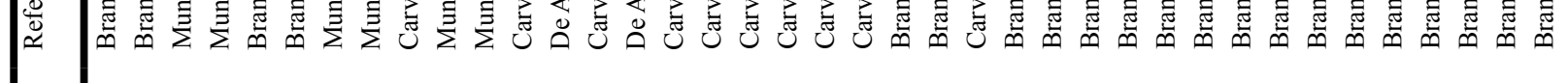

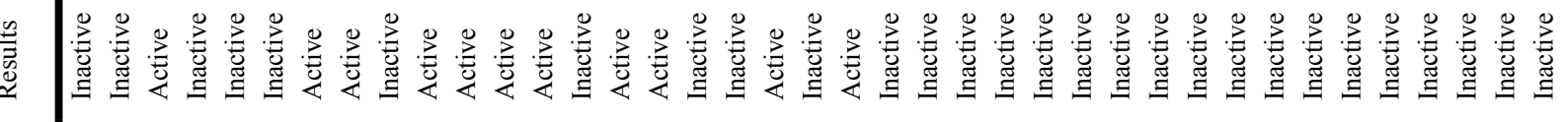

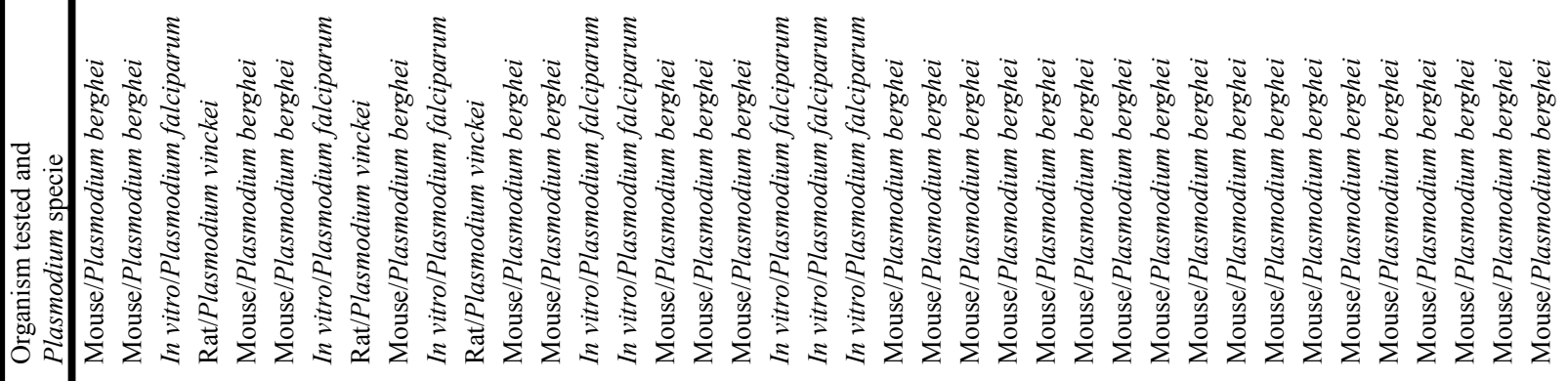

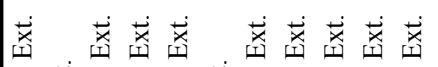

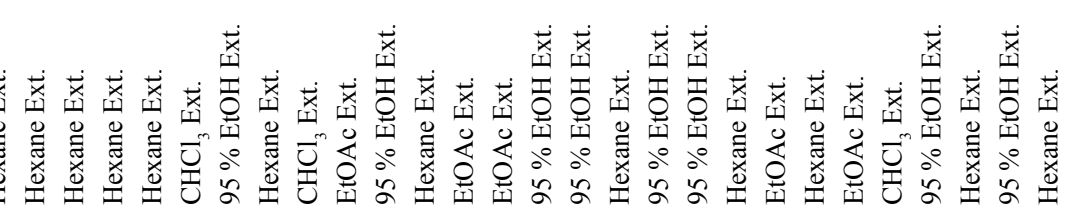

㟧泀

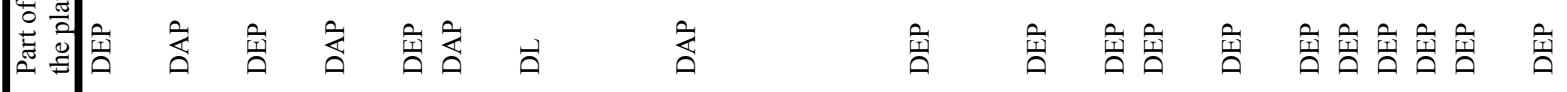

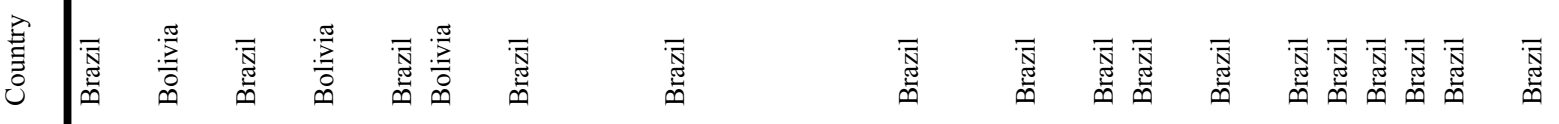

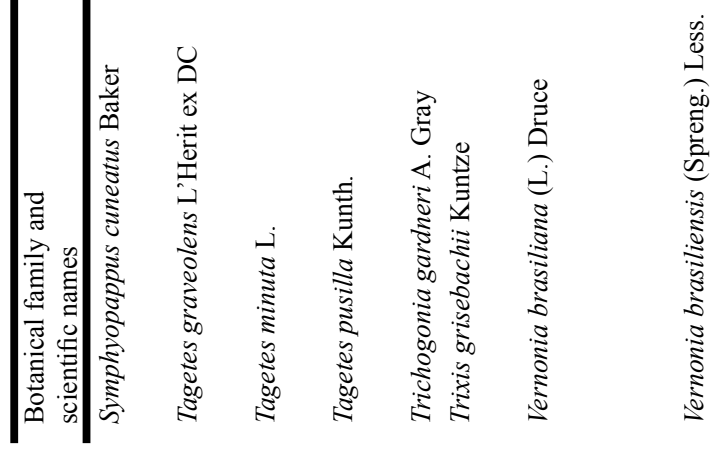

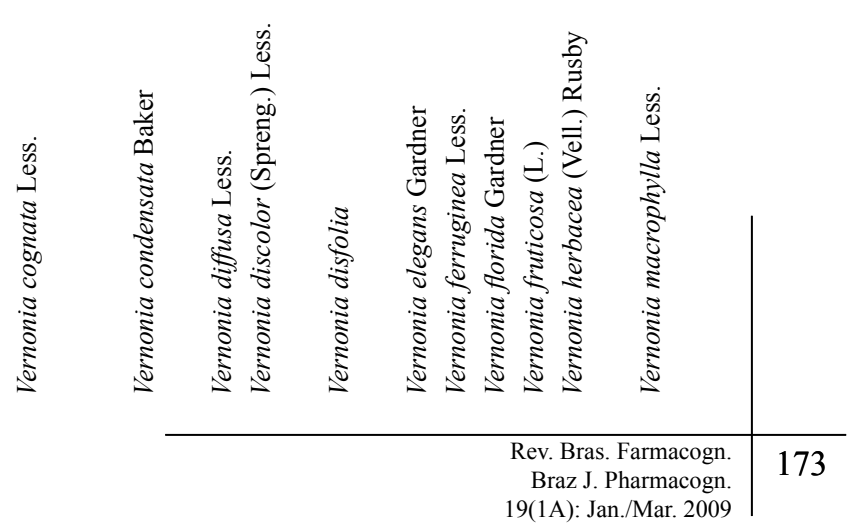




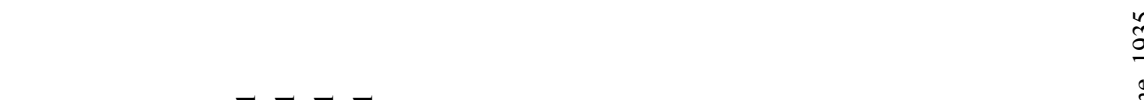

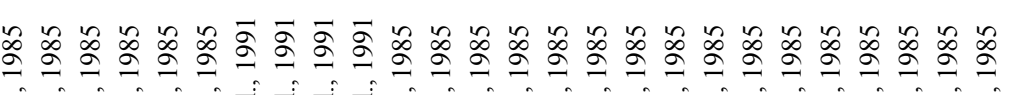

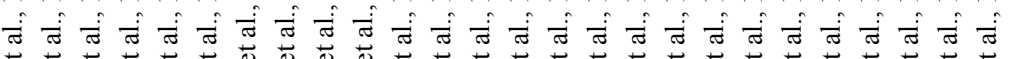

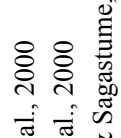

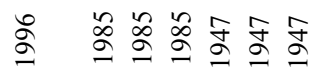

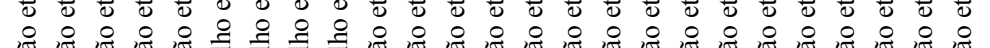

焉

कृ

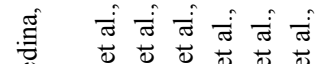

然

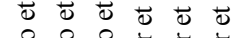

$\sum_{\text {i }}$ 选 造

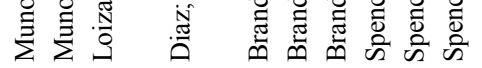

䋷

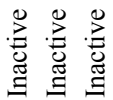

紊密

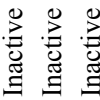

媦

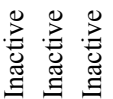

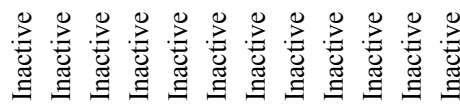

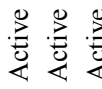

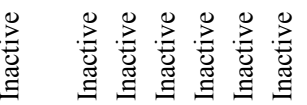

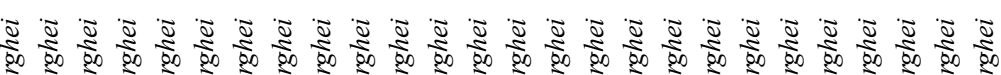

紊

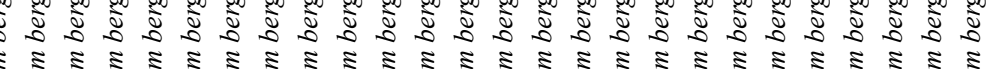

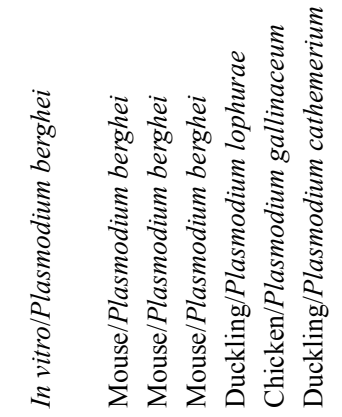

芉

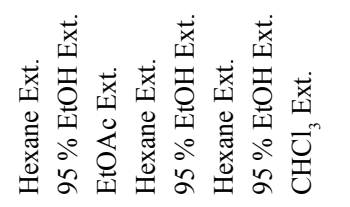




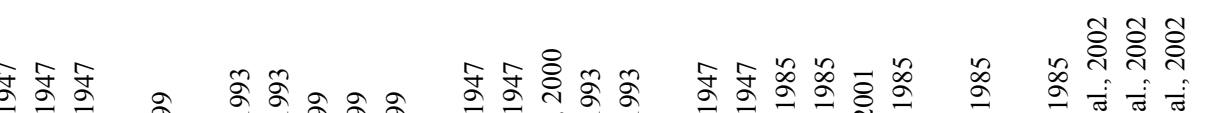

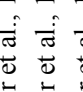

दे

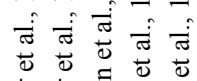

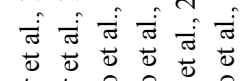

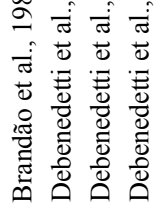

ठ্ণ

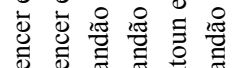

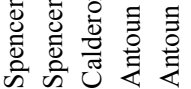

की की

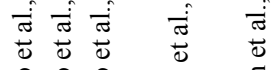

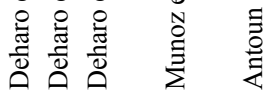

象
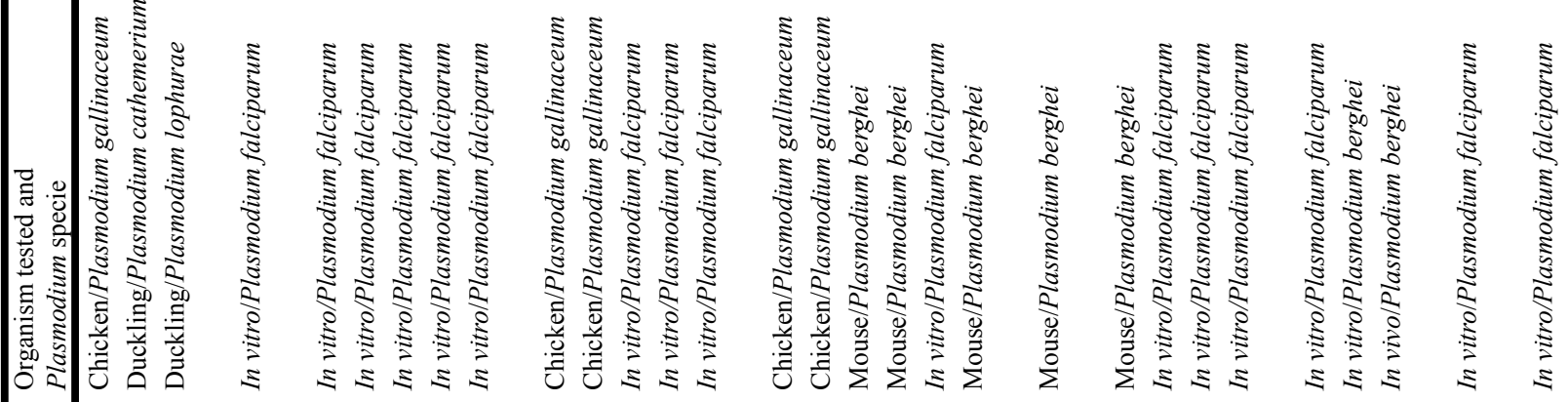

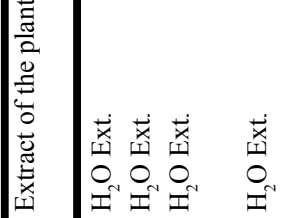

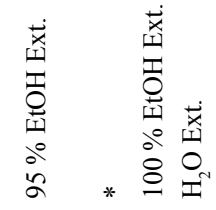

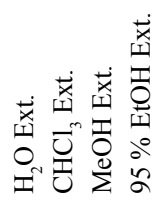

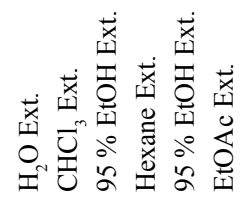

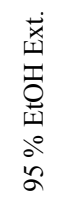

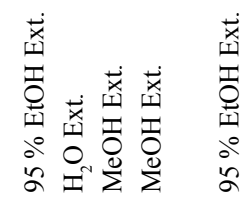

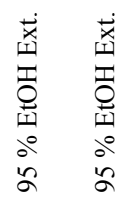

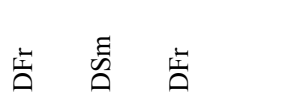

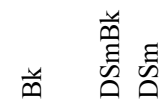

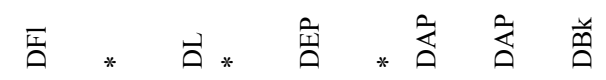

主*

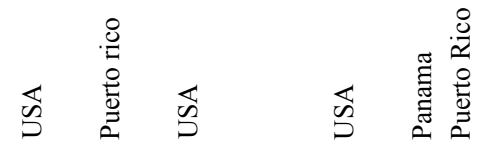

高高

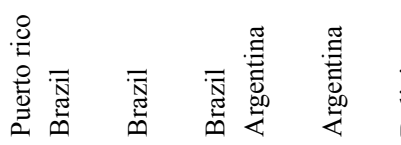

$\begin{array}{ll} & 0 \\ \sum_{0}^{\frac{0}{2}} & 0 \\ 0 & 0 \\ 0 & 0 \\ 0 & 0 \\ 0\end{array}$
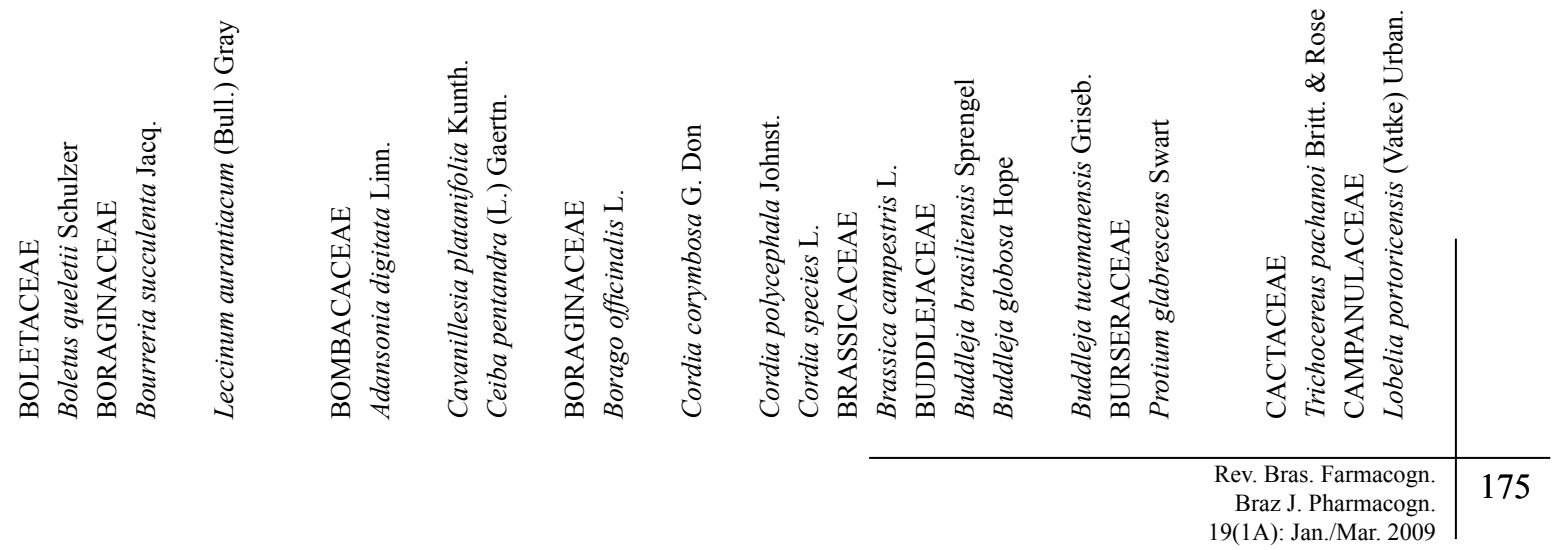


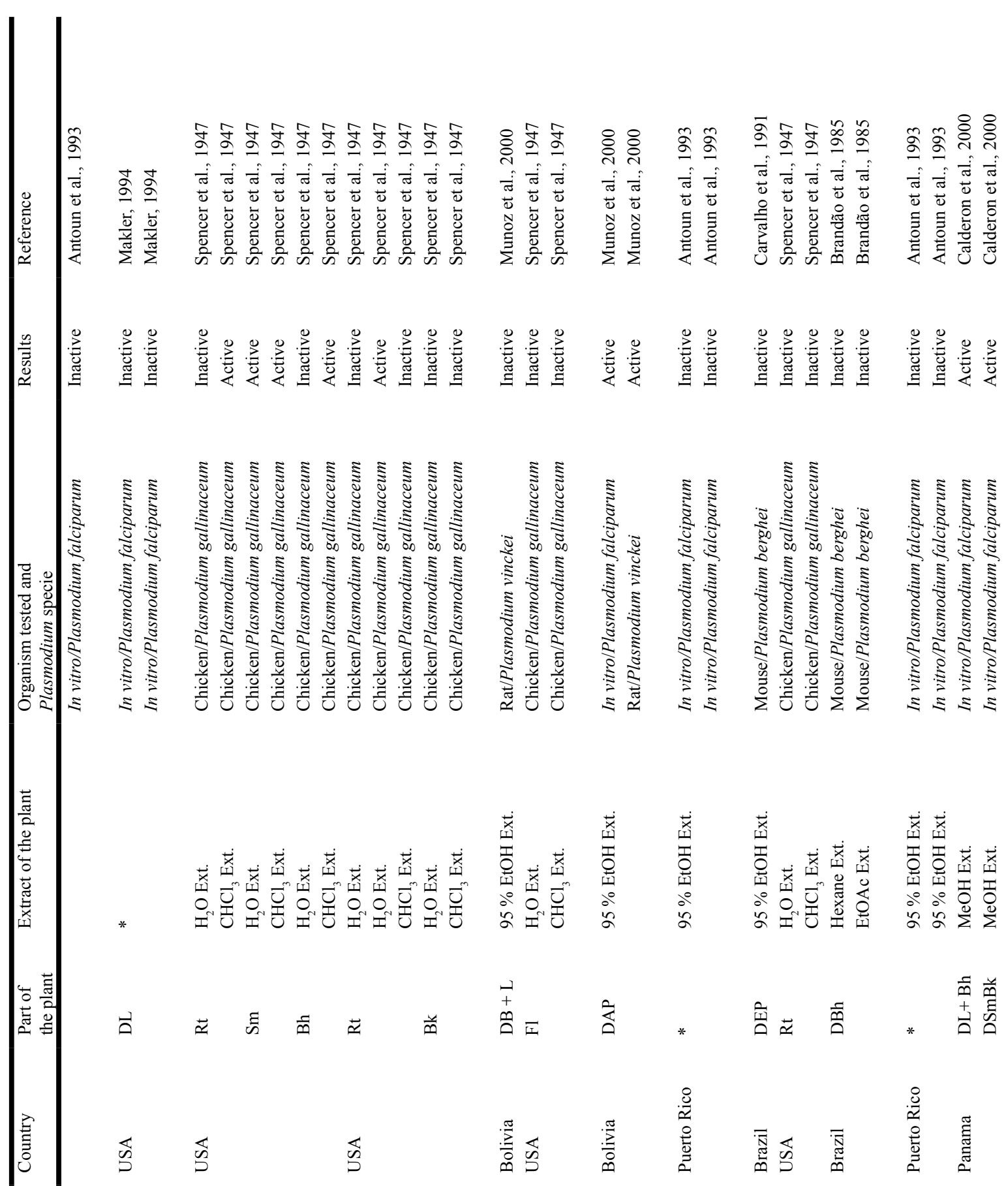


ঃ $\overline{\mathrm{\sigma}} \overline{\mathrm{\sigma}} \overline{\mathrm{\sigma}} \mathrm{g}$

$\bar{\partial} \overline{\mathrm{a}}$

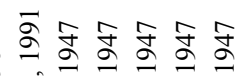

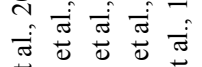

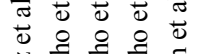

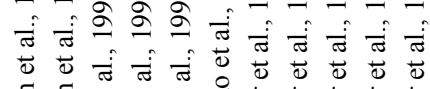

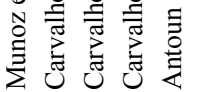

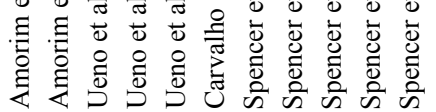

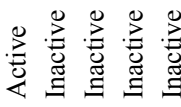

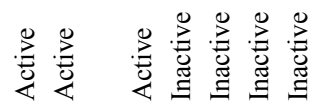

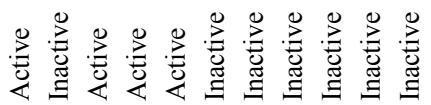

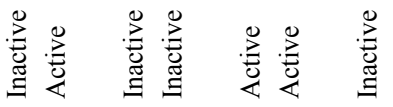

ำ
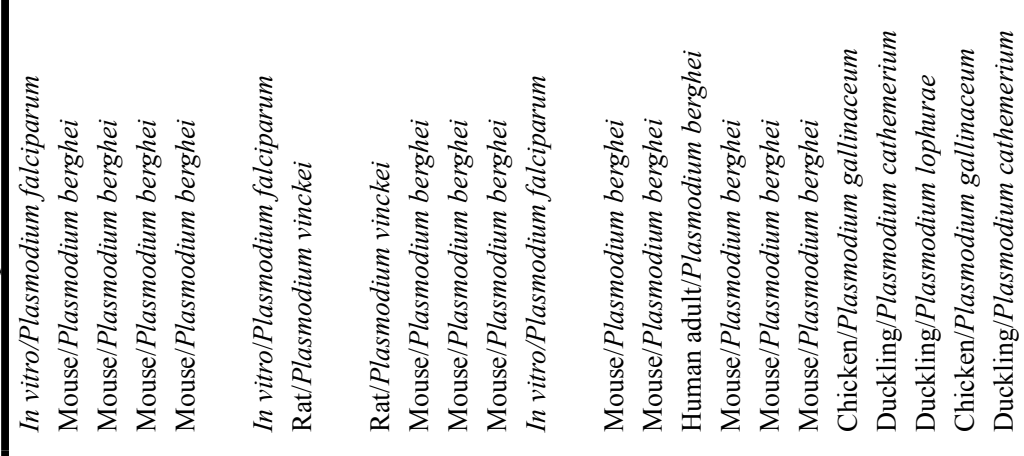

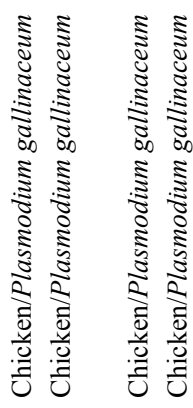

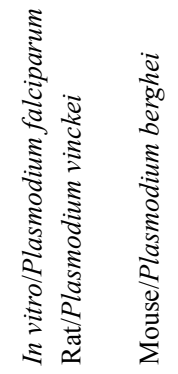

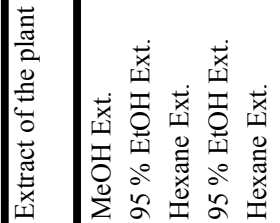

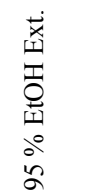

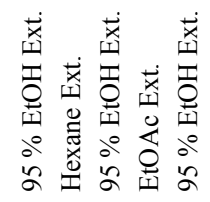

蓠蓄蓄

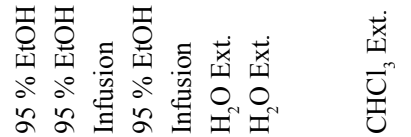

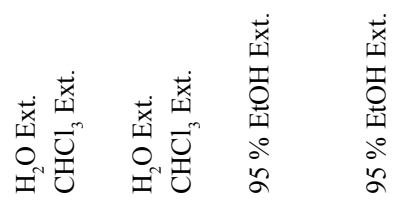

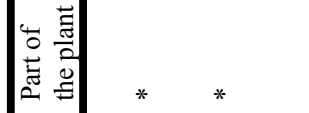

育

定定

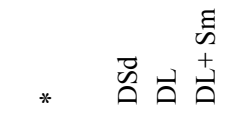

压主

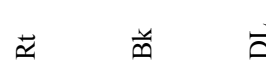

ถี

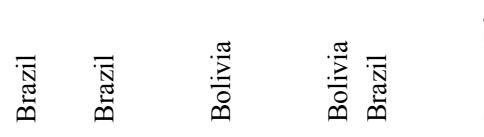

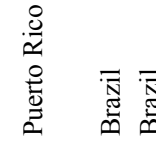

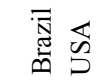

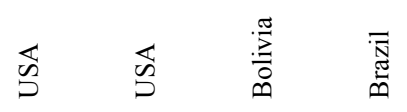

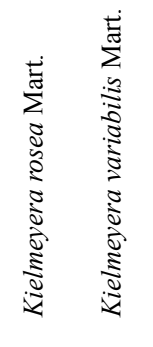
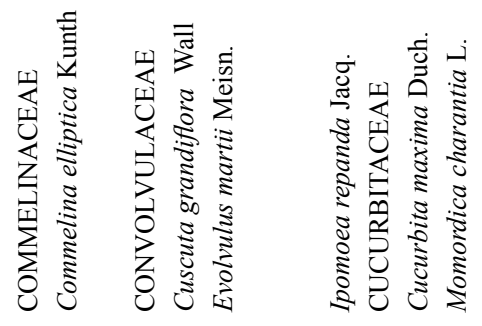

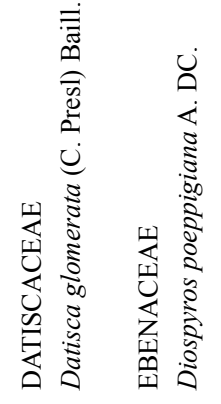

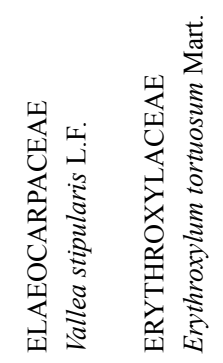




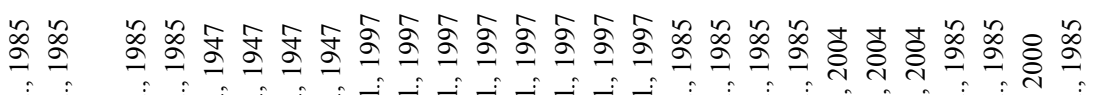

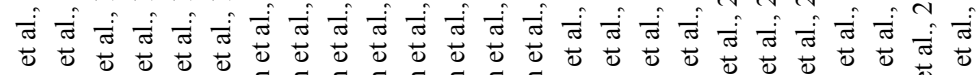

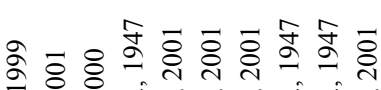
这

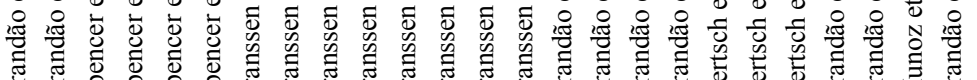

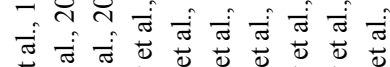

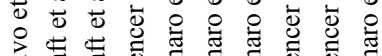

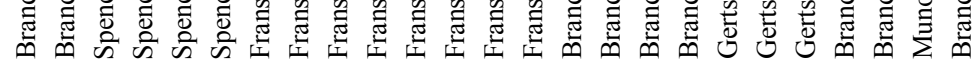

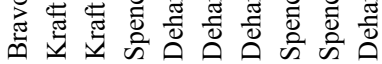

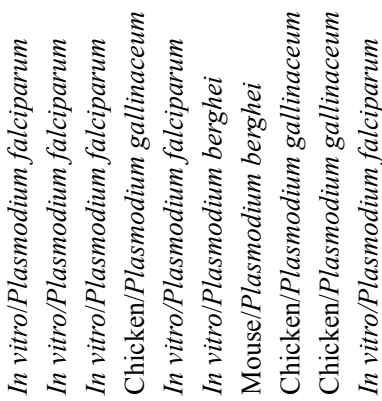

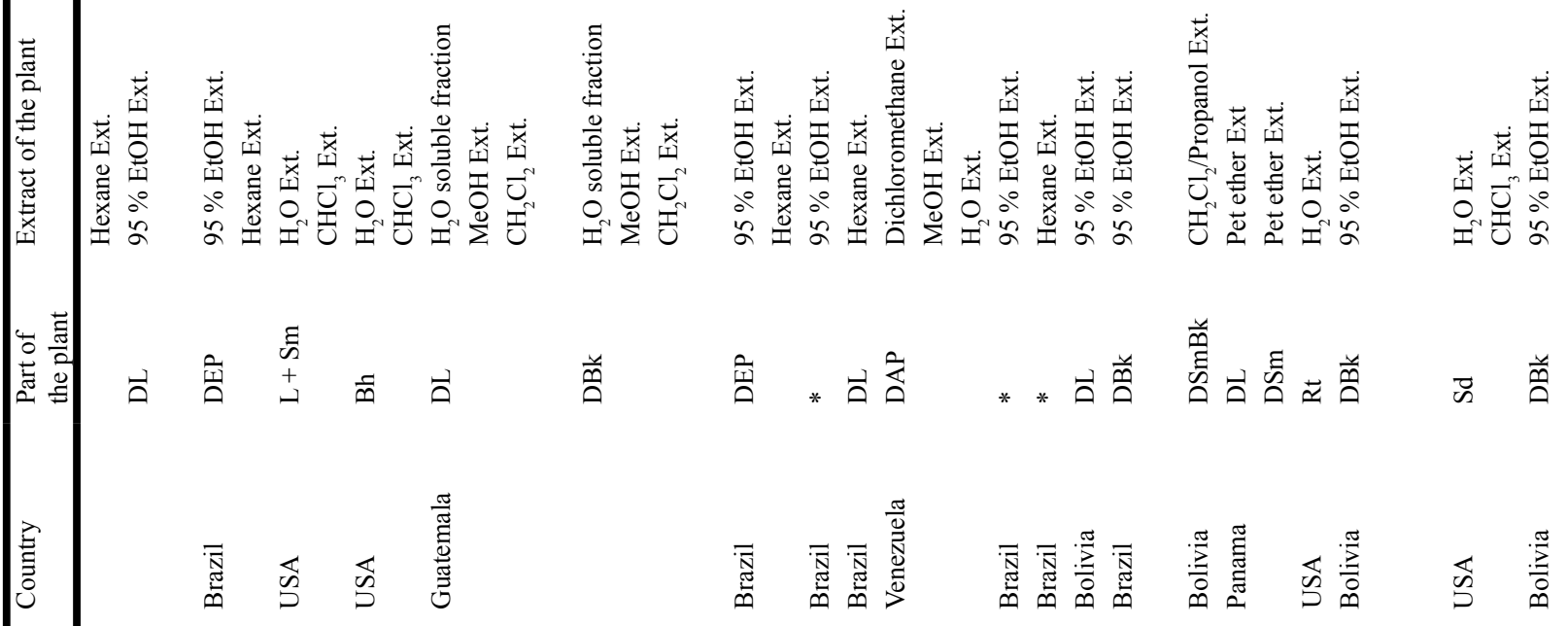




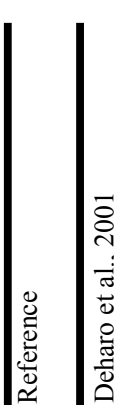

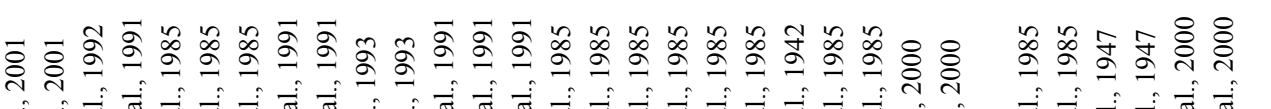

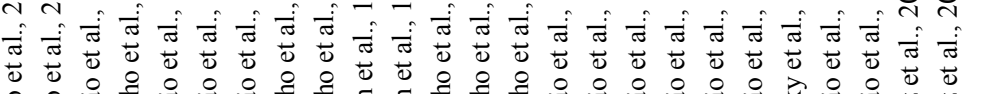

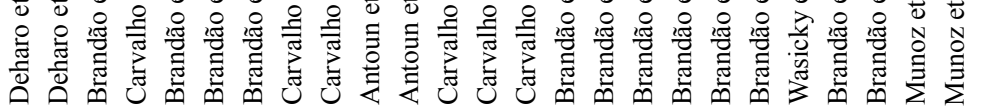

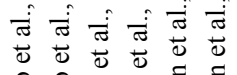

ڤे ڤे ठे

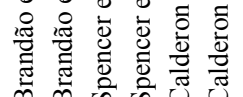

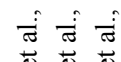

ڤิণ สं $\frac{0}{\pi} \frac{0}{4}$

वे क्षे

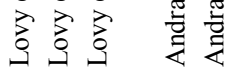

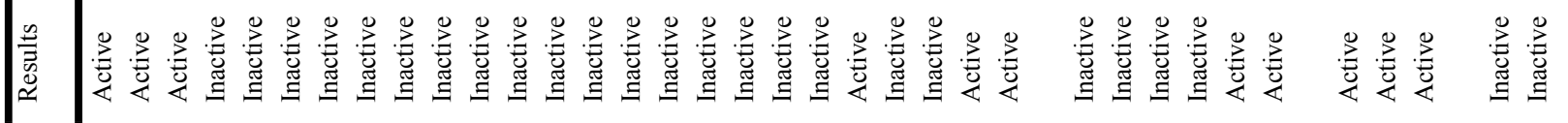

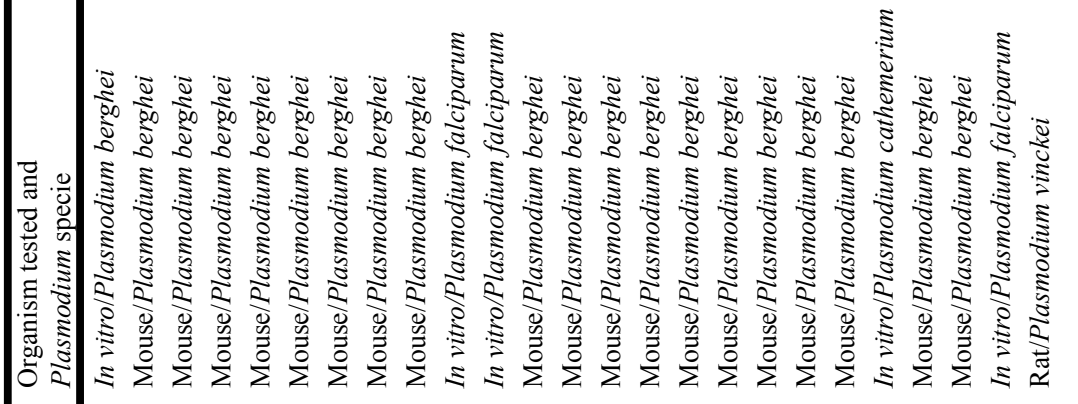
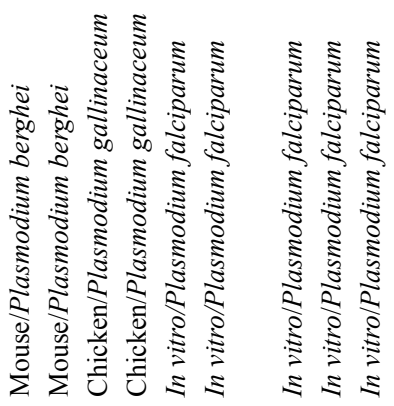

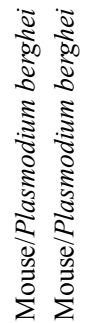

茫

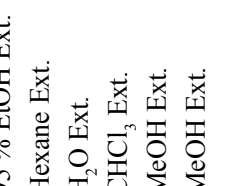

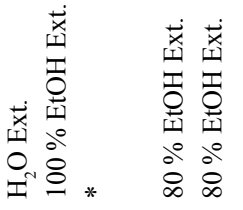
落 $\overrightarrow{\tilde{a}}$ 藏

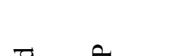

$$
\text { . }
$$

$\begin{array}{lll}\overline{\widetilde{N}} & \overline{\mathrm{N}} & \overline{\mathrm{W}} \\ \text { ต } & & \end{array}$

量

高高

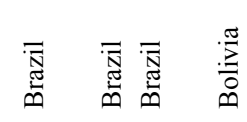

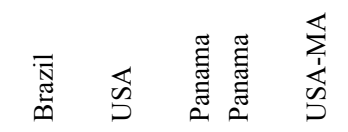

$\overline{\bar{N}}$

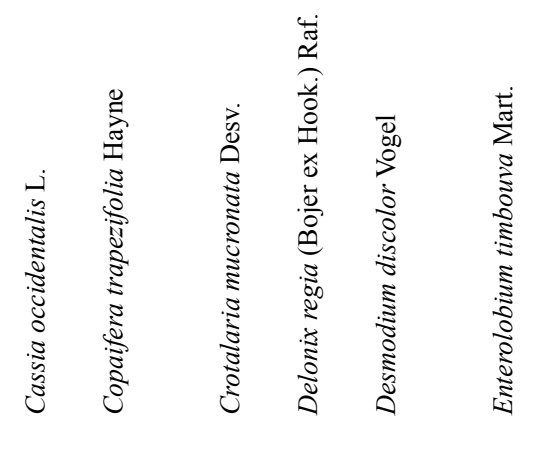

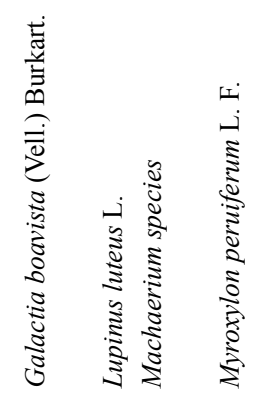

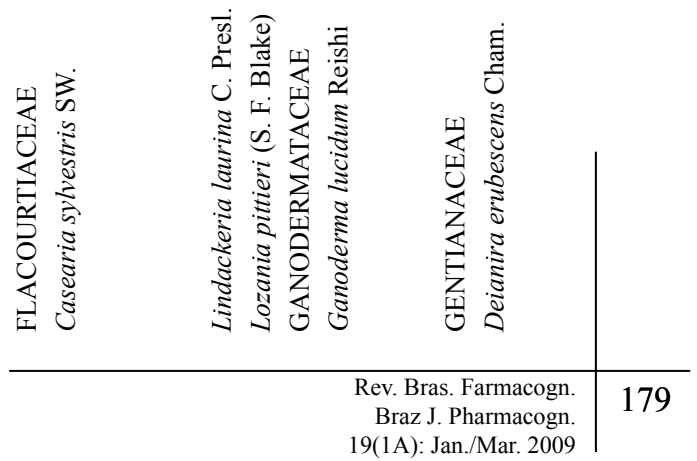


|

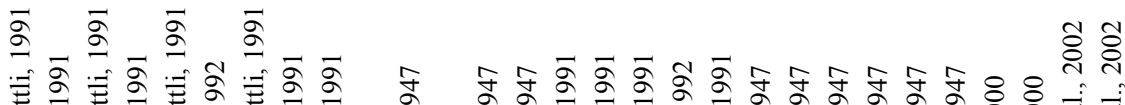

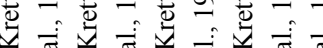

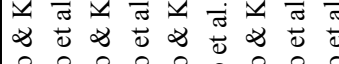

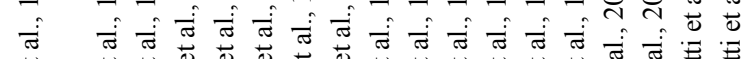

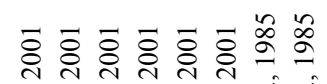

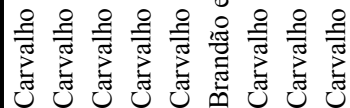

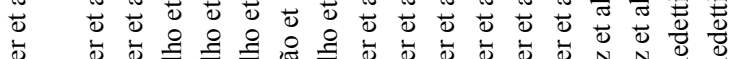

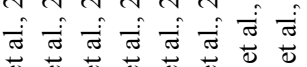

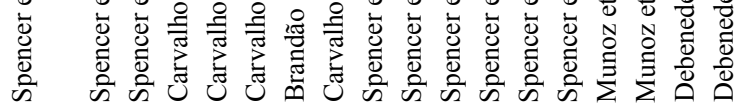

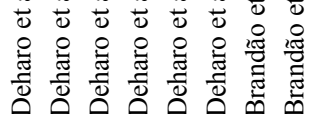

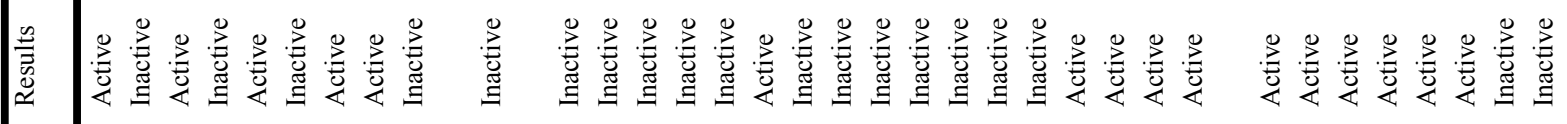

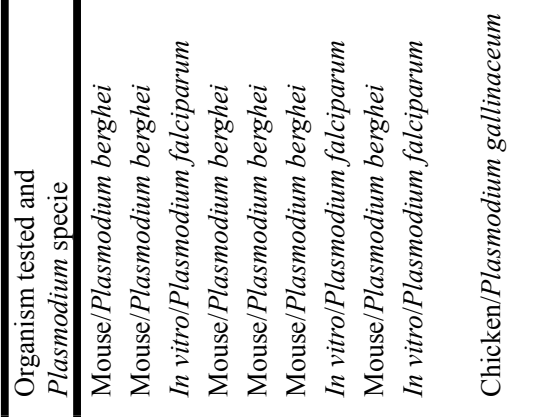

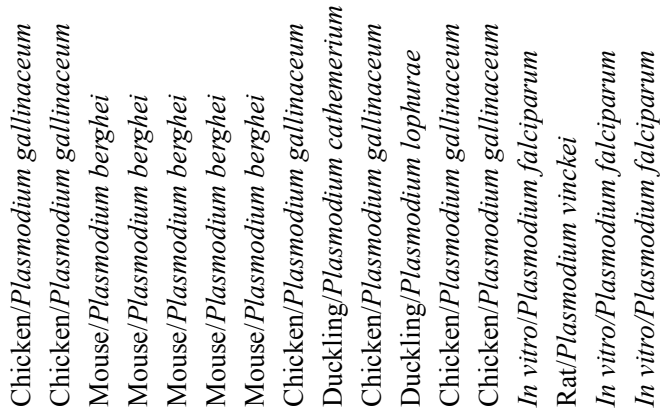

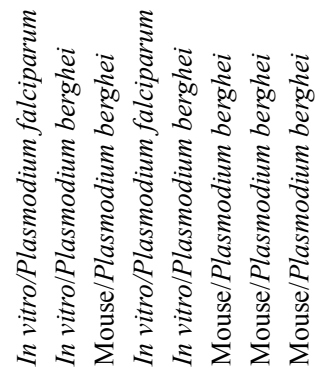
专

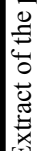
这

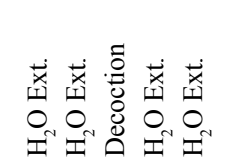
荠

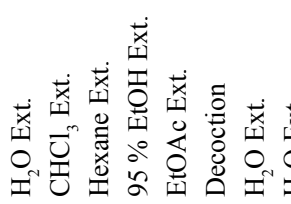
蓠

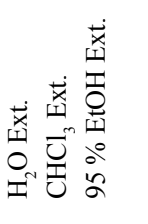

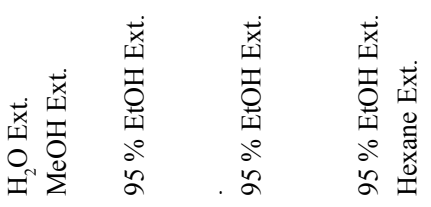
능 㐘 


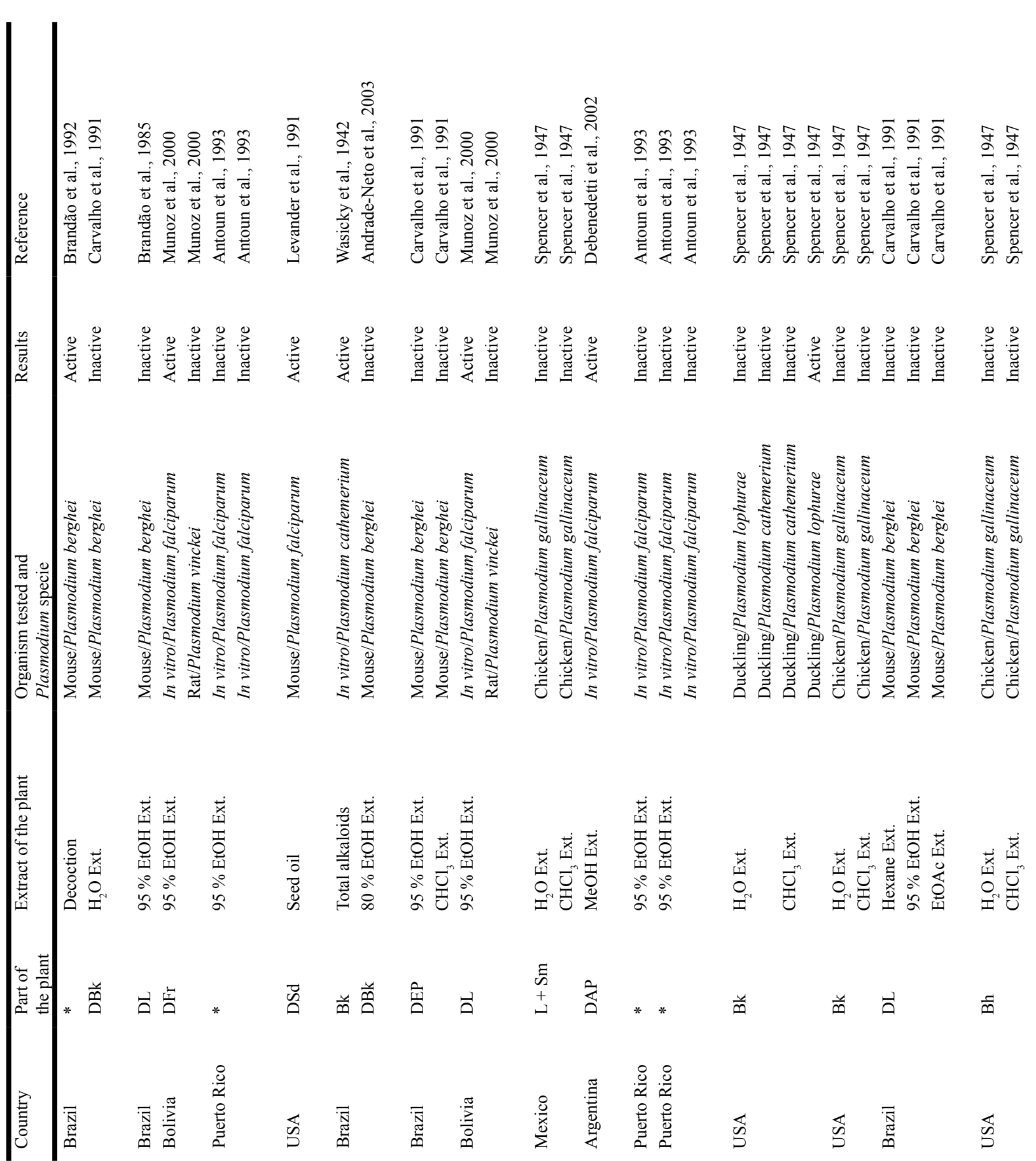




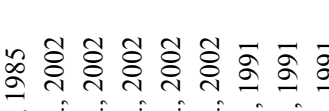

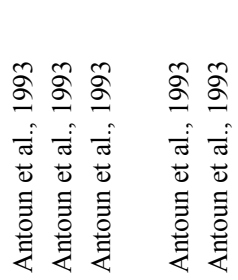

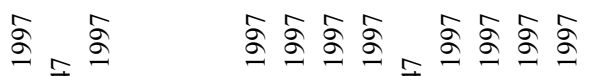

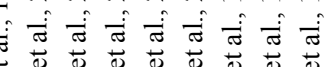

ลे

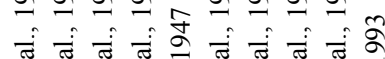

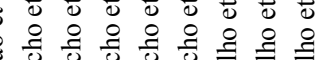

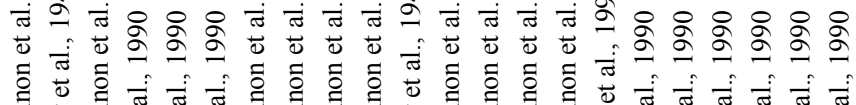

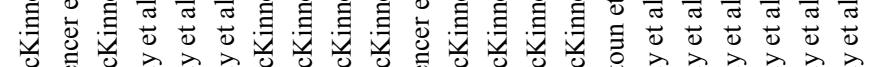

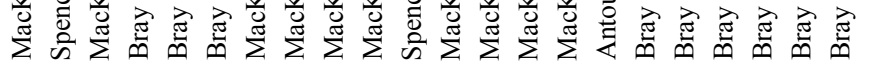

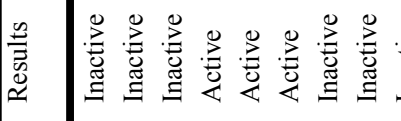

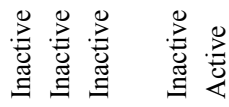

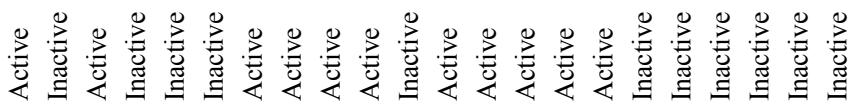

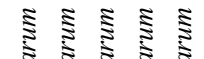

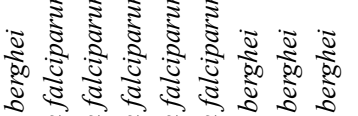

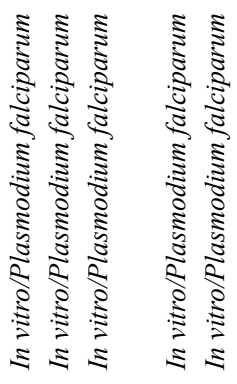

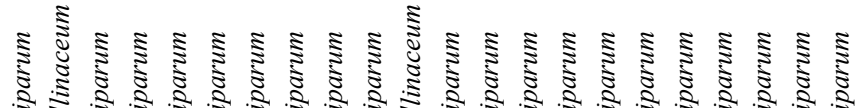

0
0
0

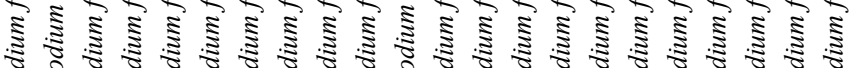

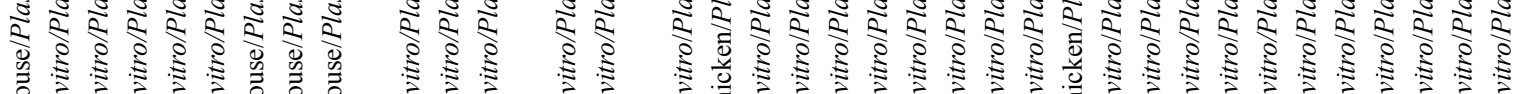

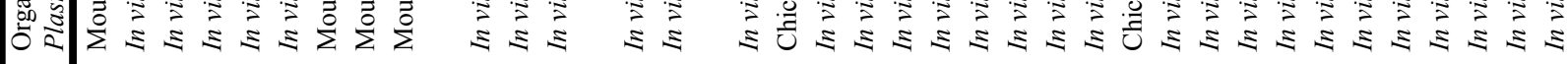

蒙

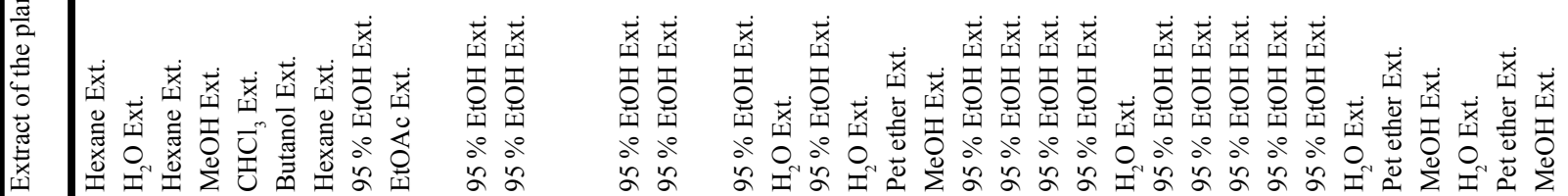

xis

㟧泀

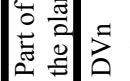

产 $* * *$ *

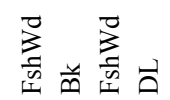

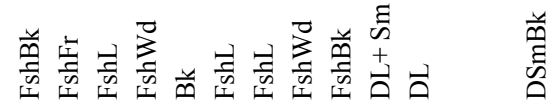

U

気

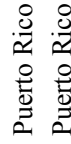

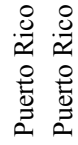

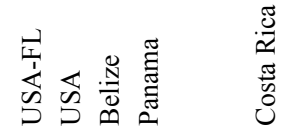

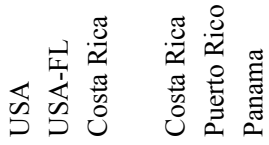

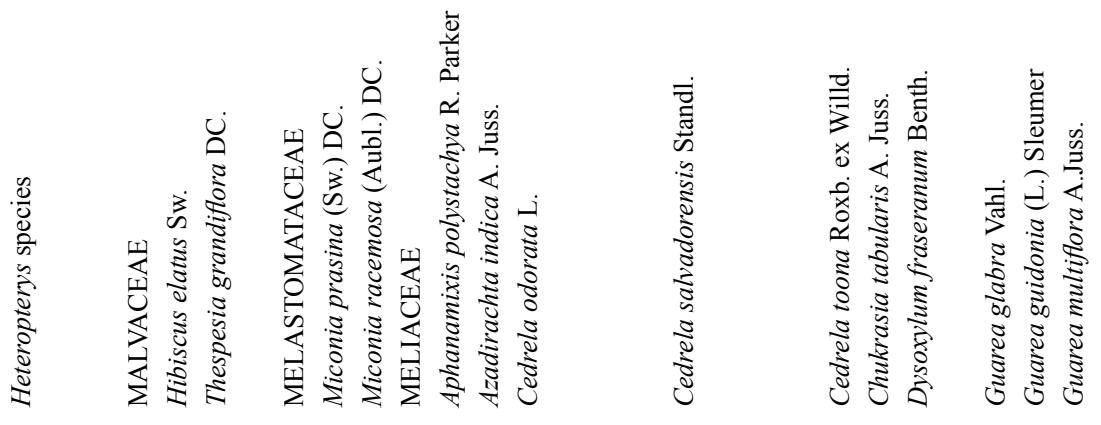




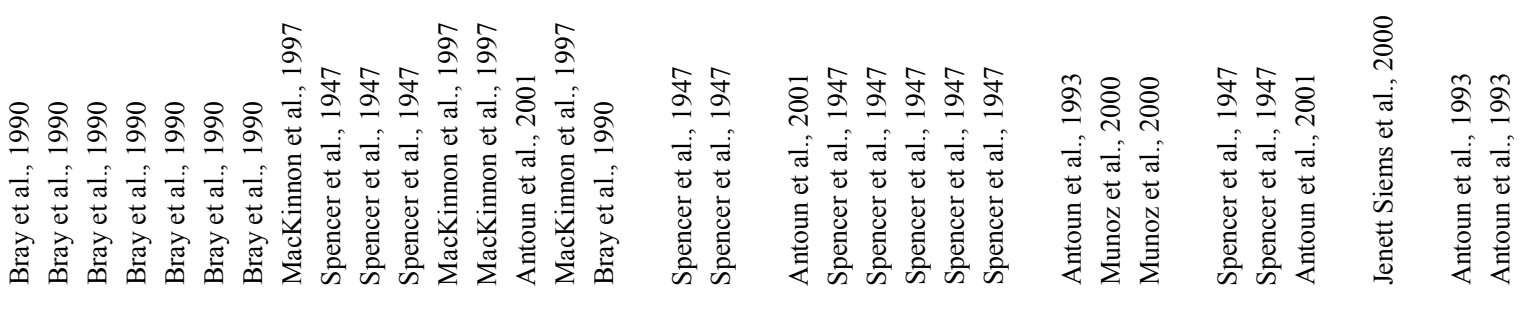

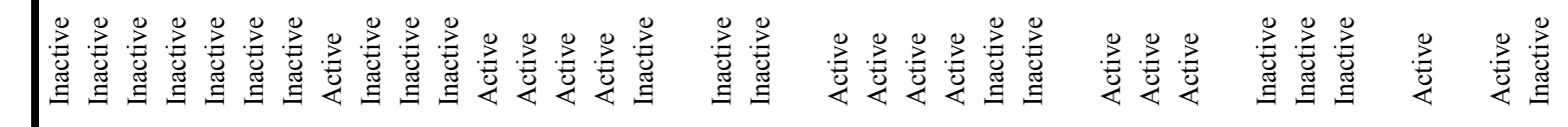

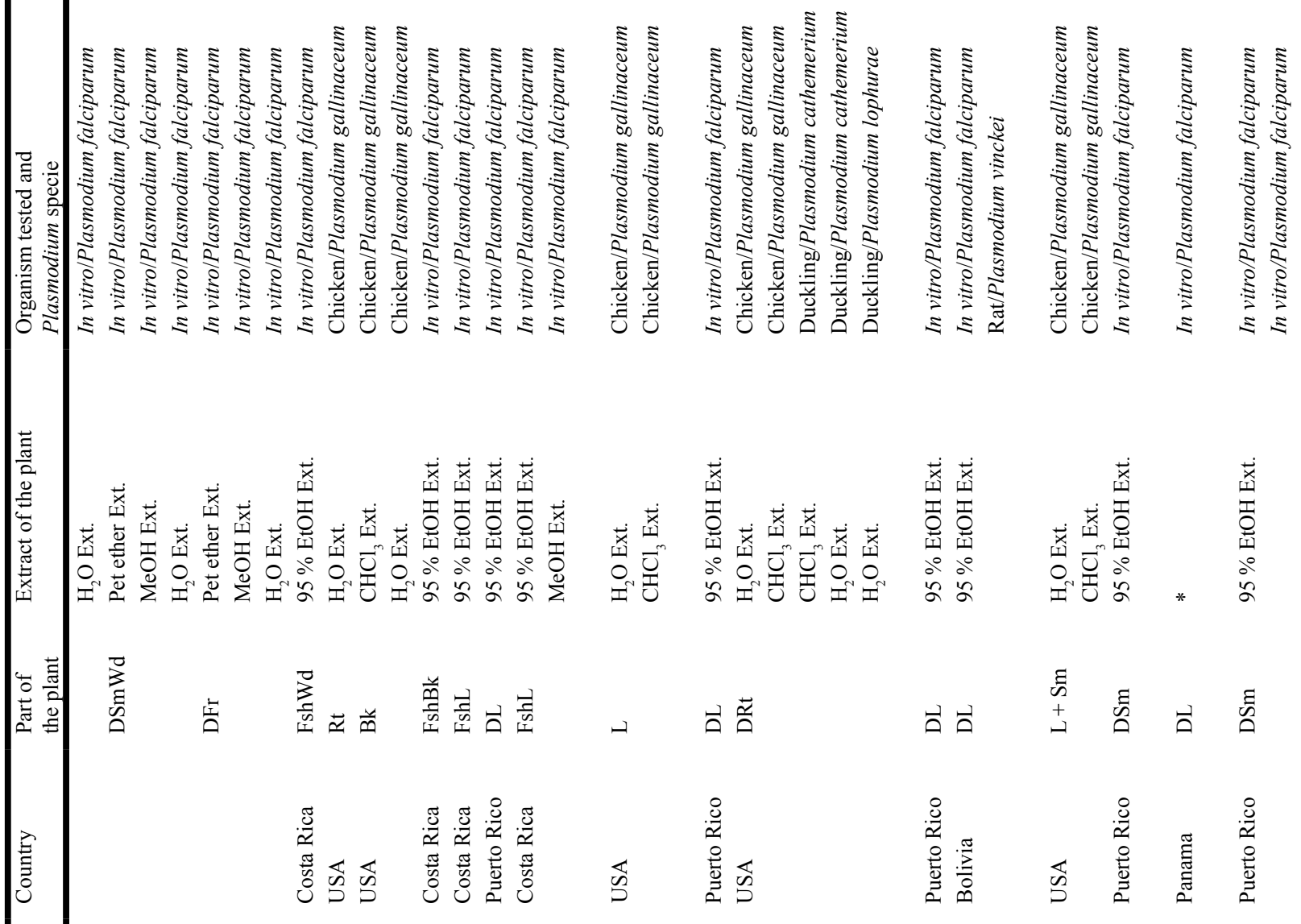
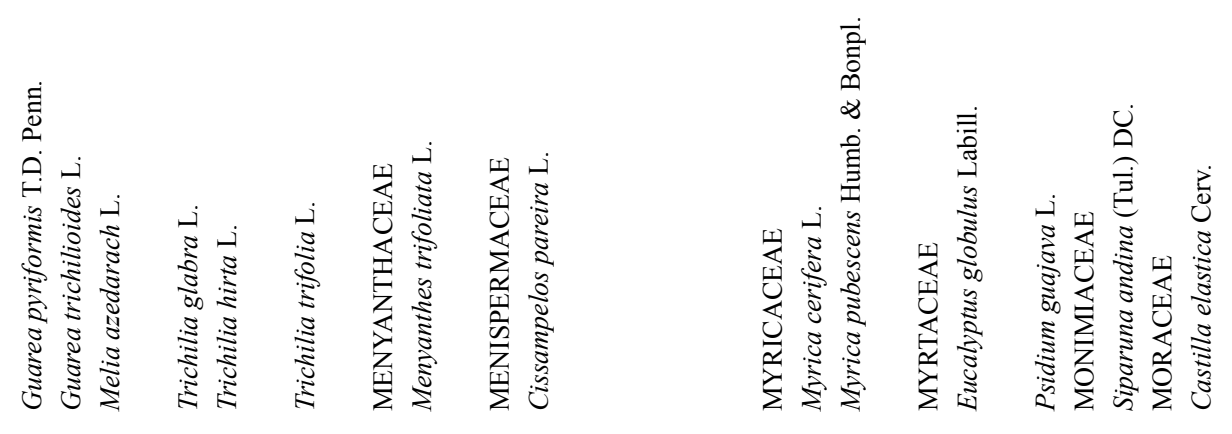


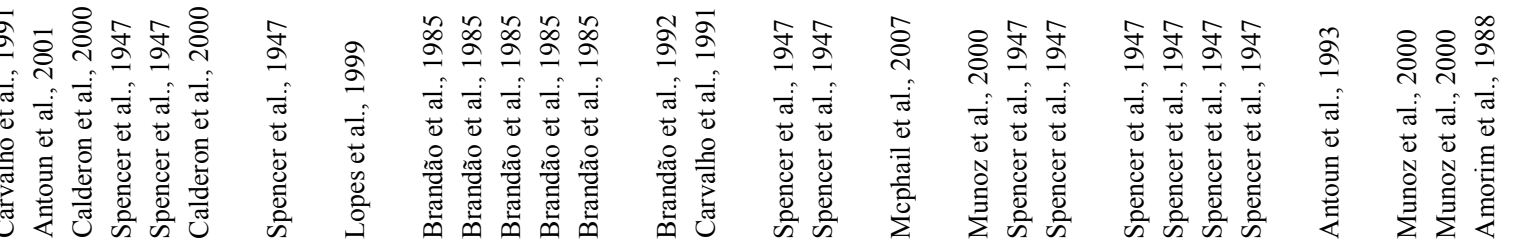

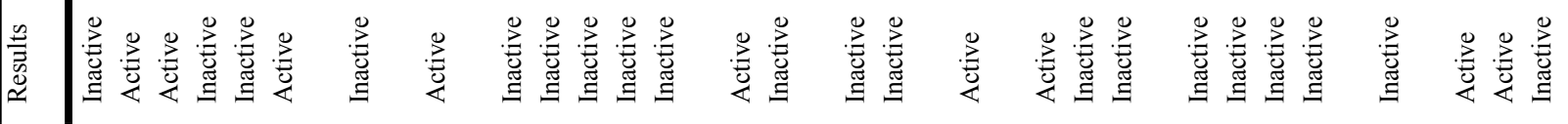

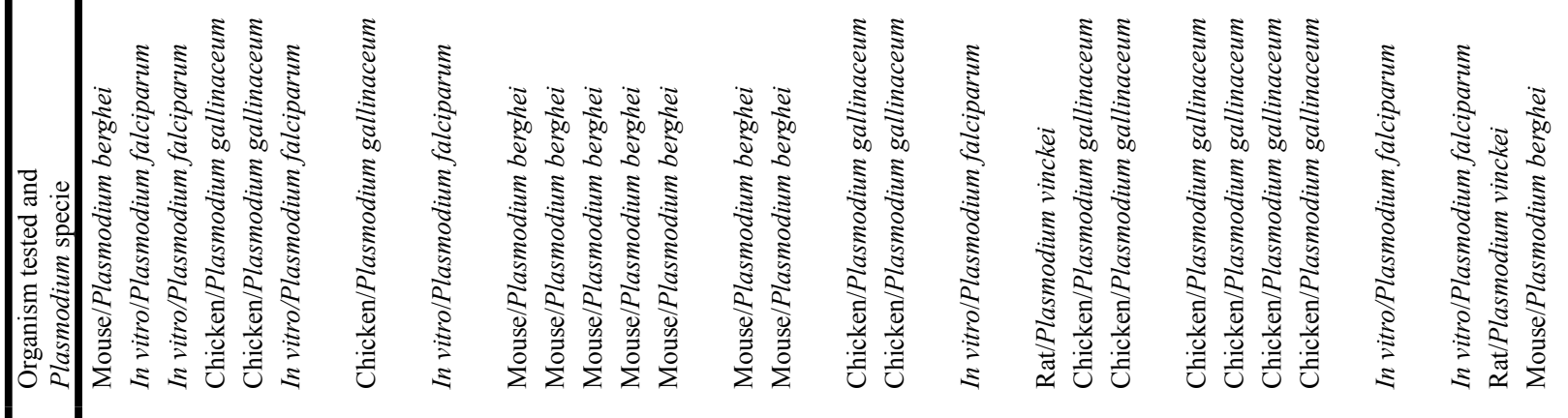

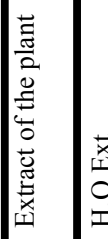

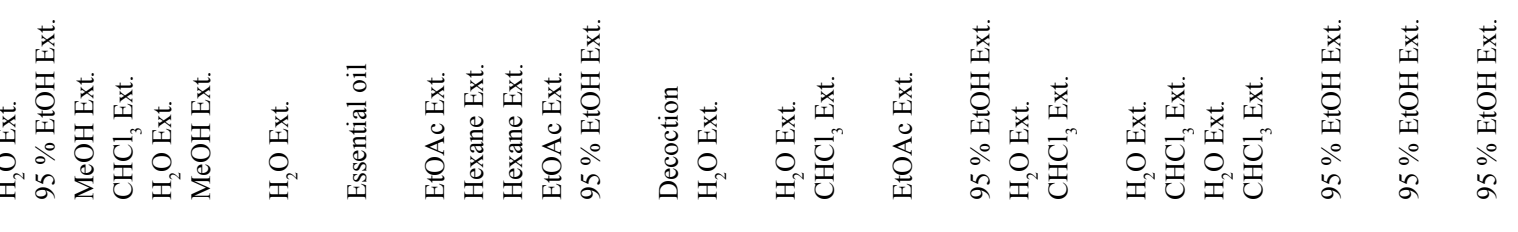

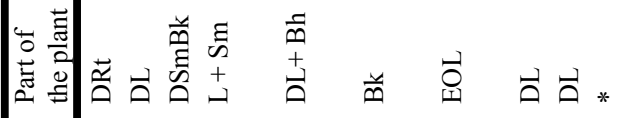

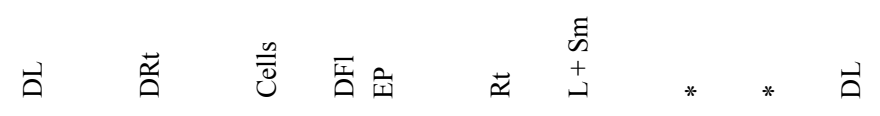

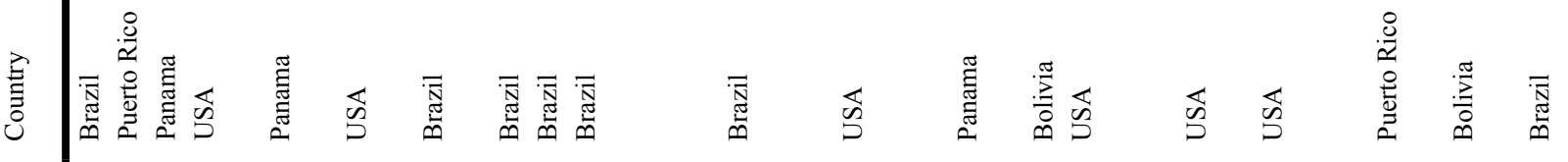

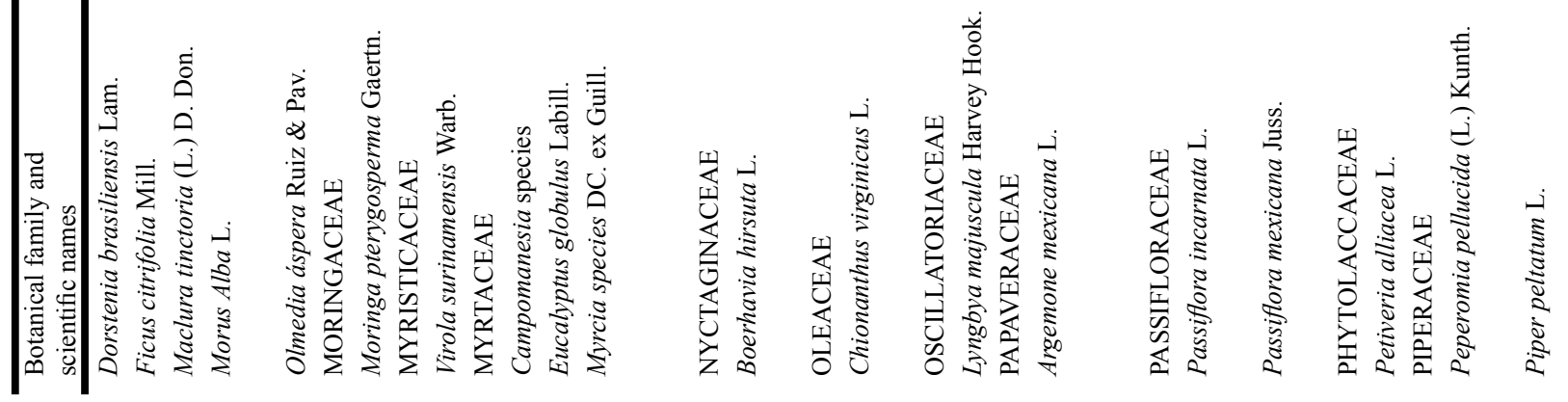



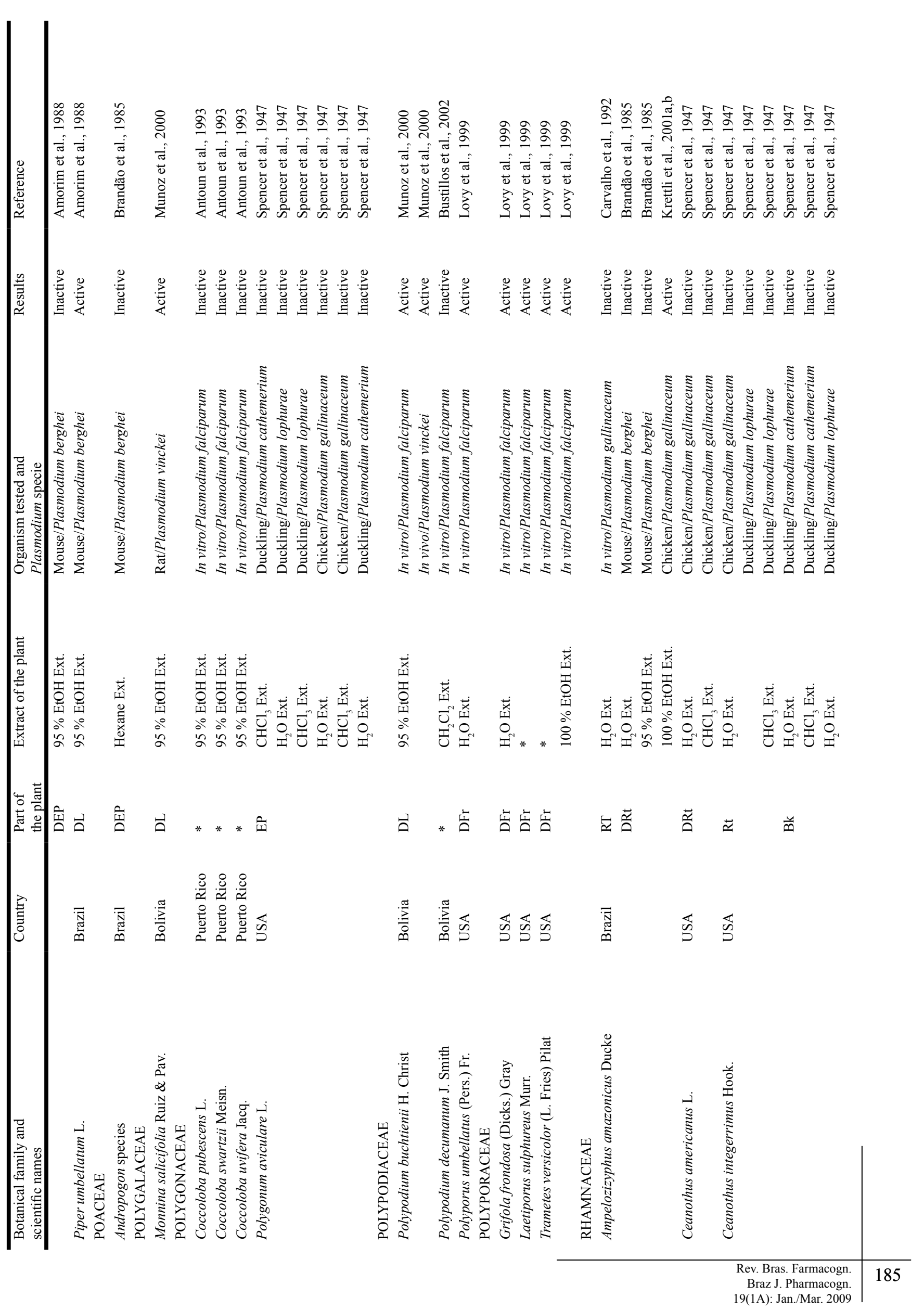
$\stackrel{n}{2}$

$\stackrel{n}{2}$

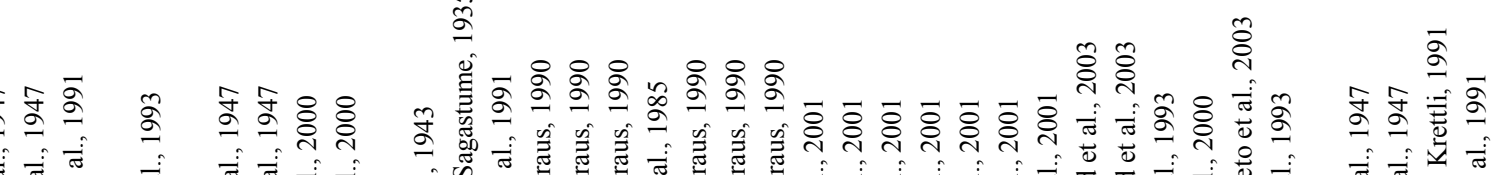

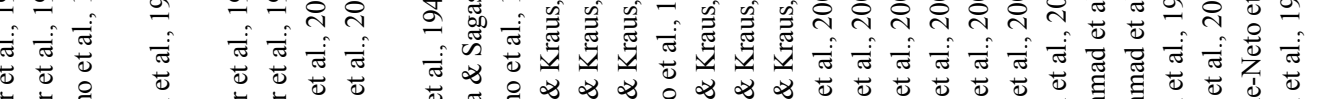

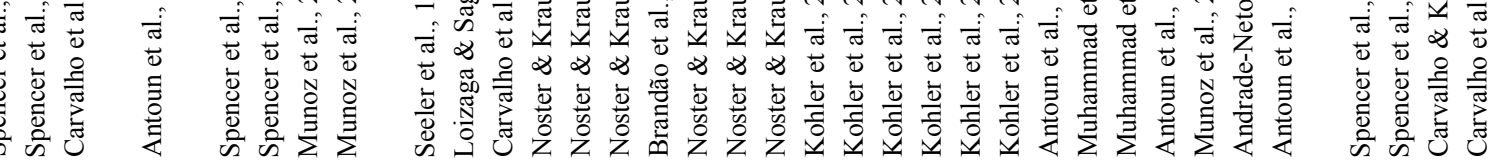

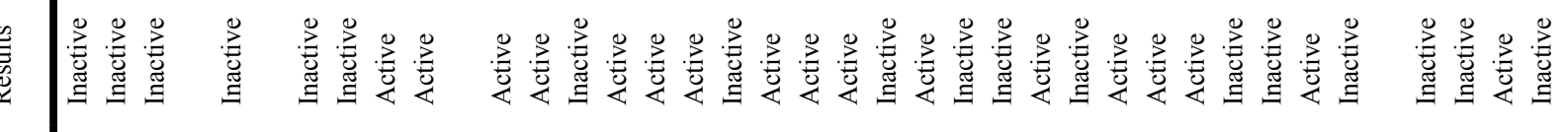

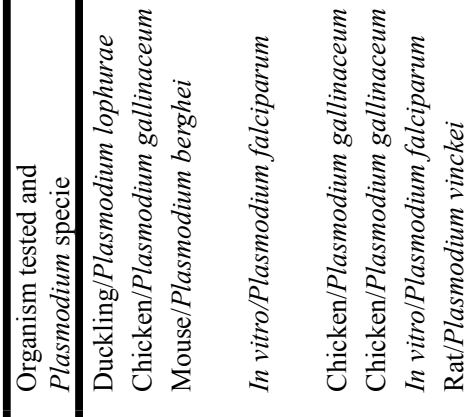
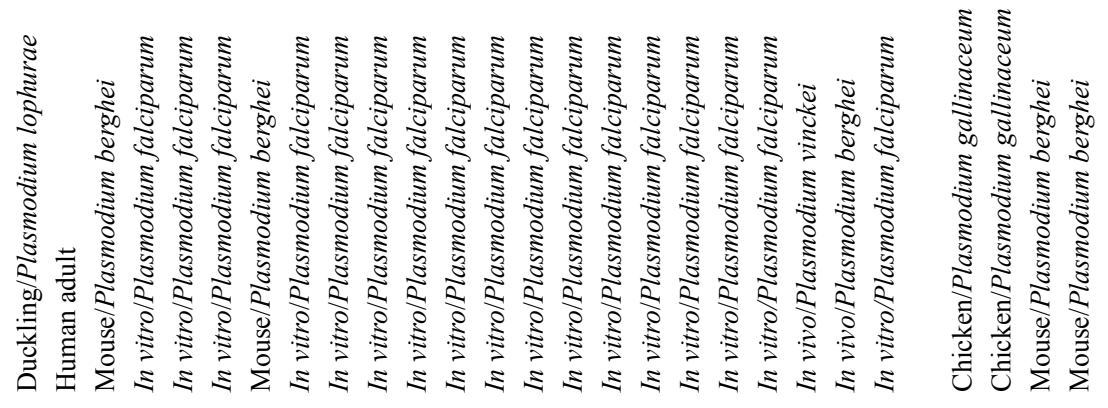

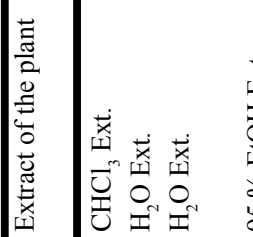

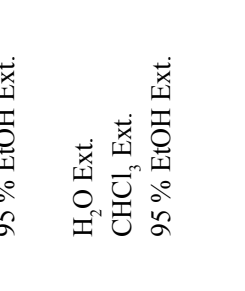

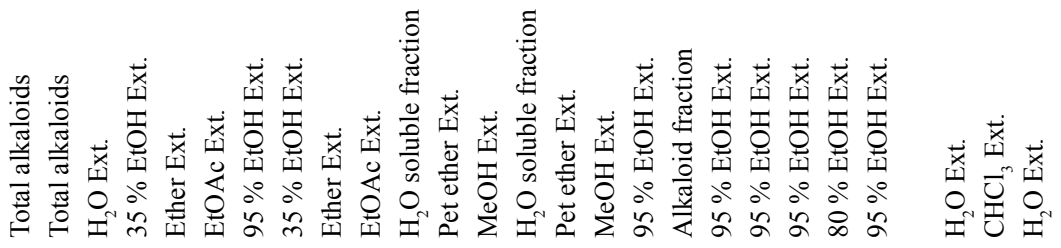

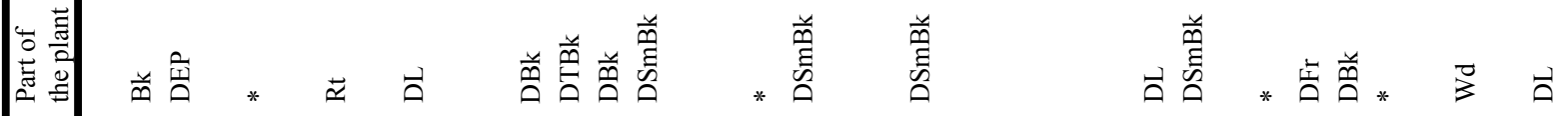

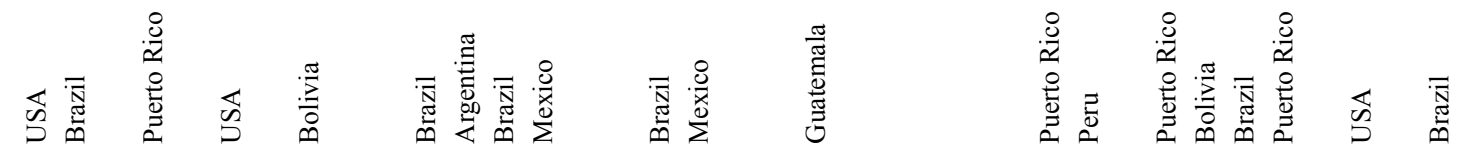

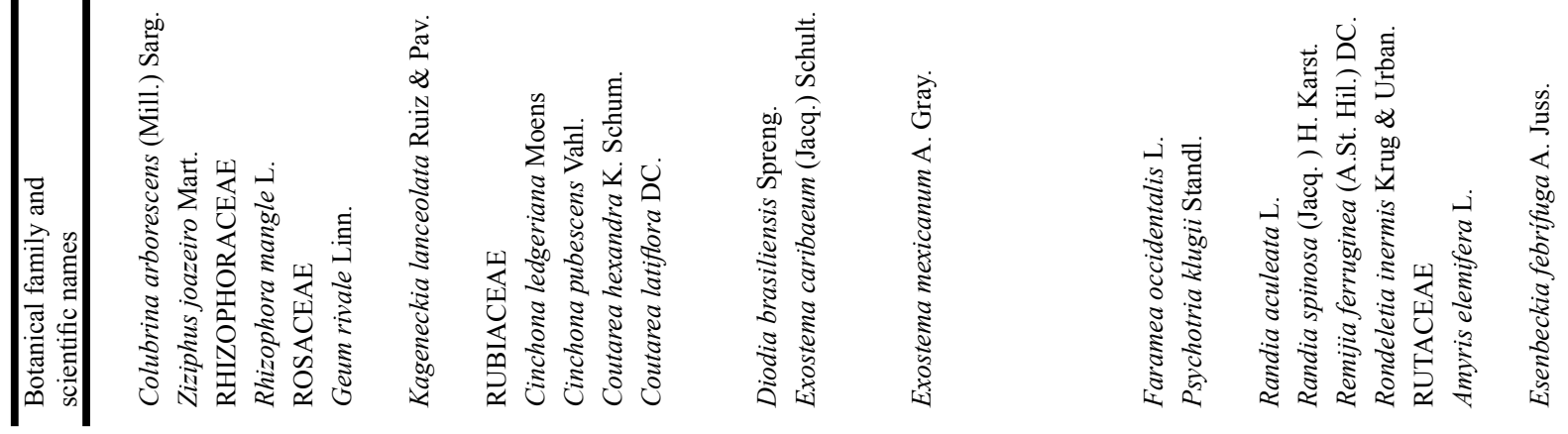




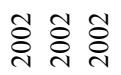

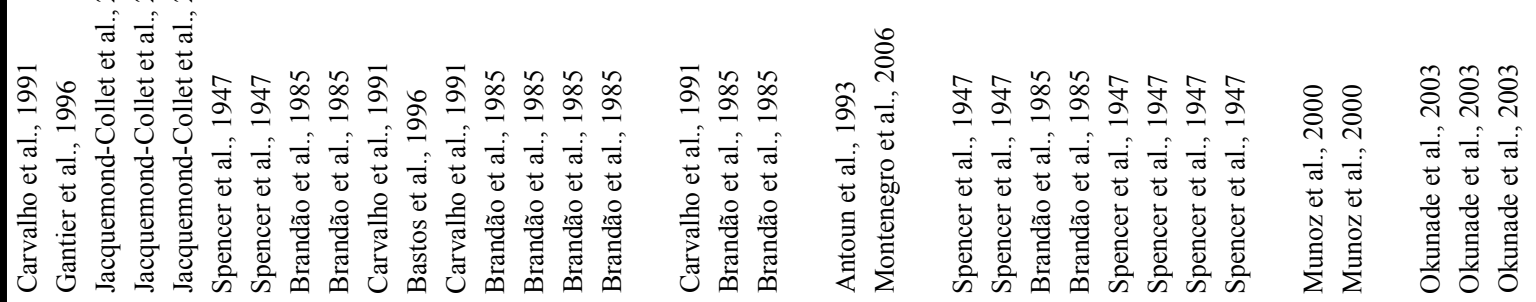

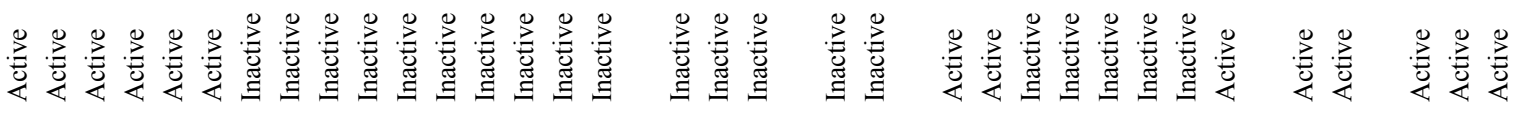

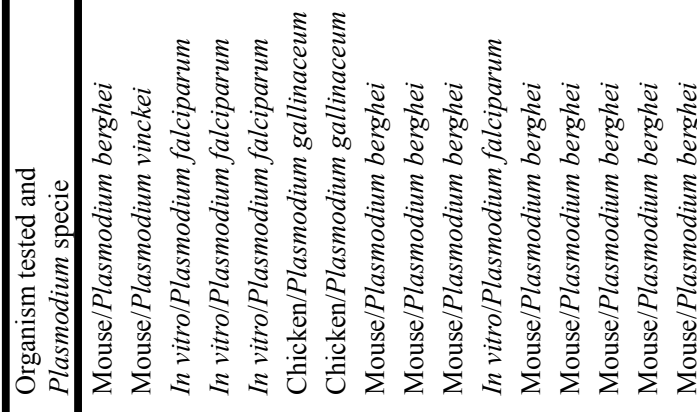
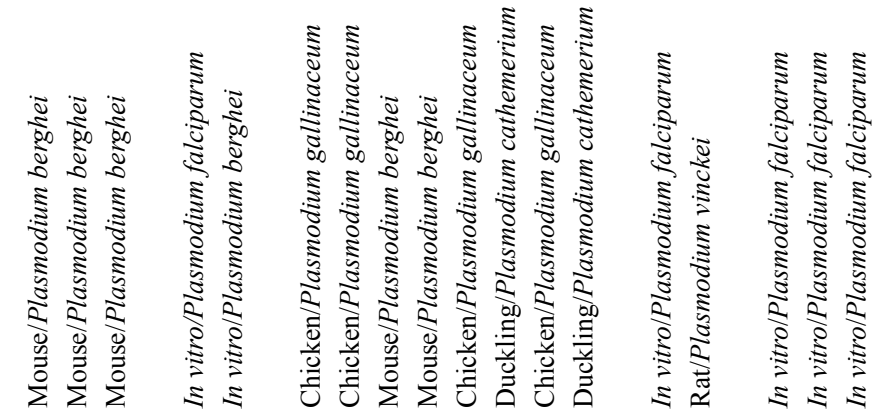

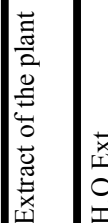

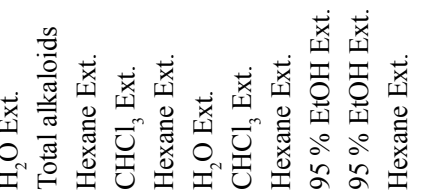

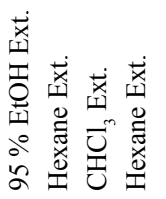

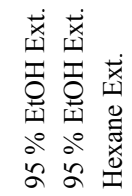

蓠蓄

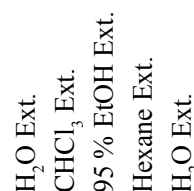

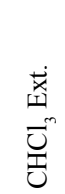

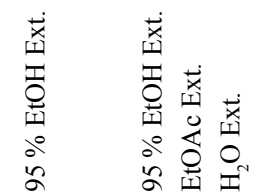

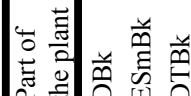

㕗

$\ddot{\simeq}+\overrightarrow{0}$

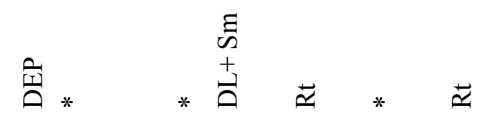

芒部

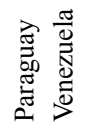

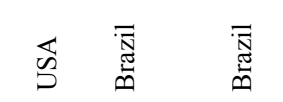

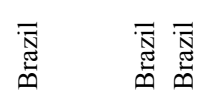

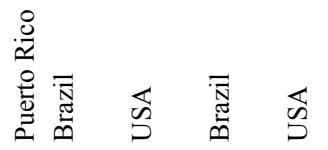

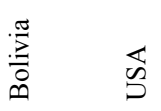

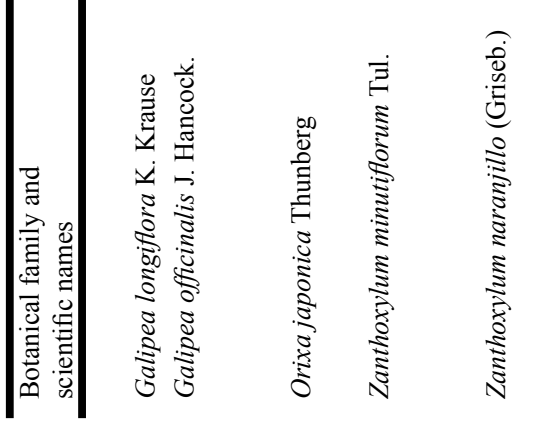
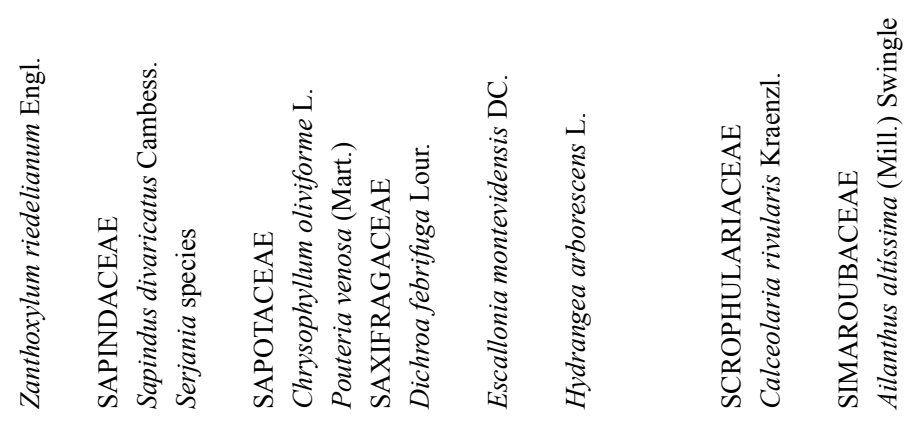


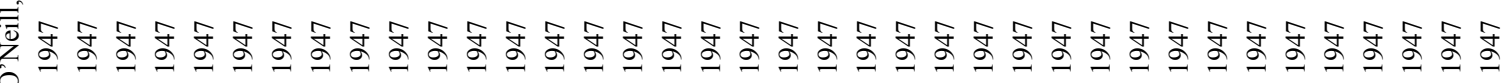

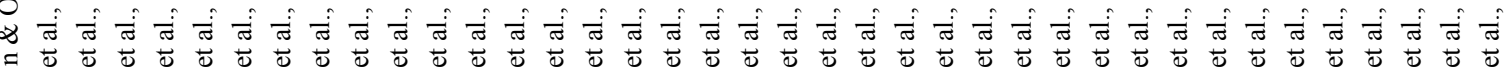

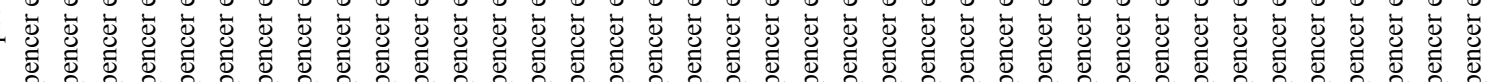

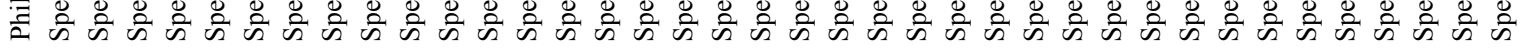

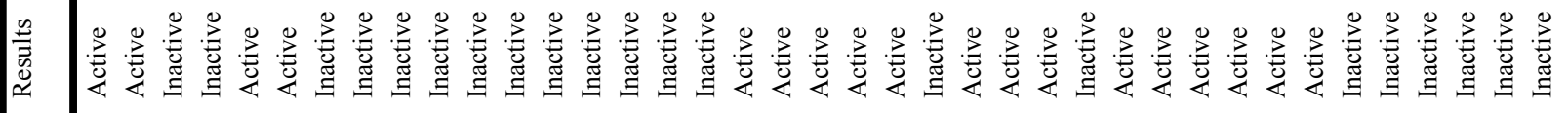

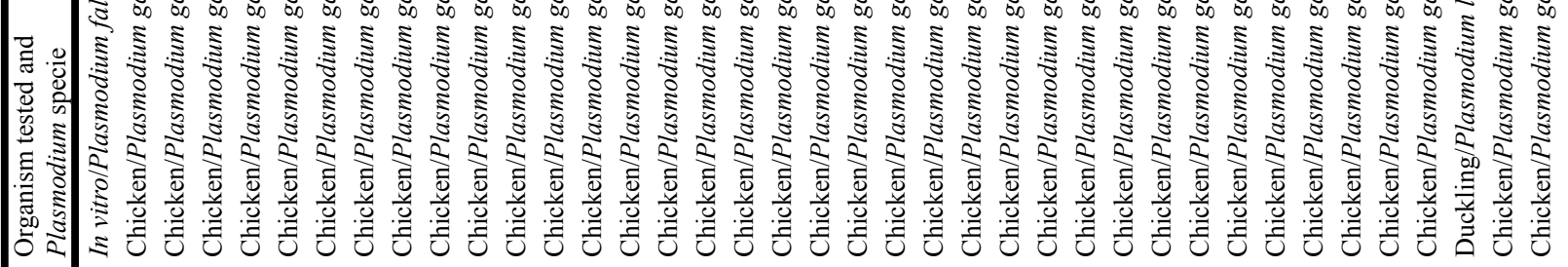

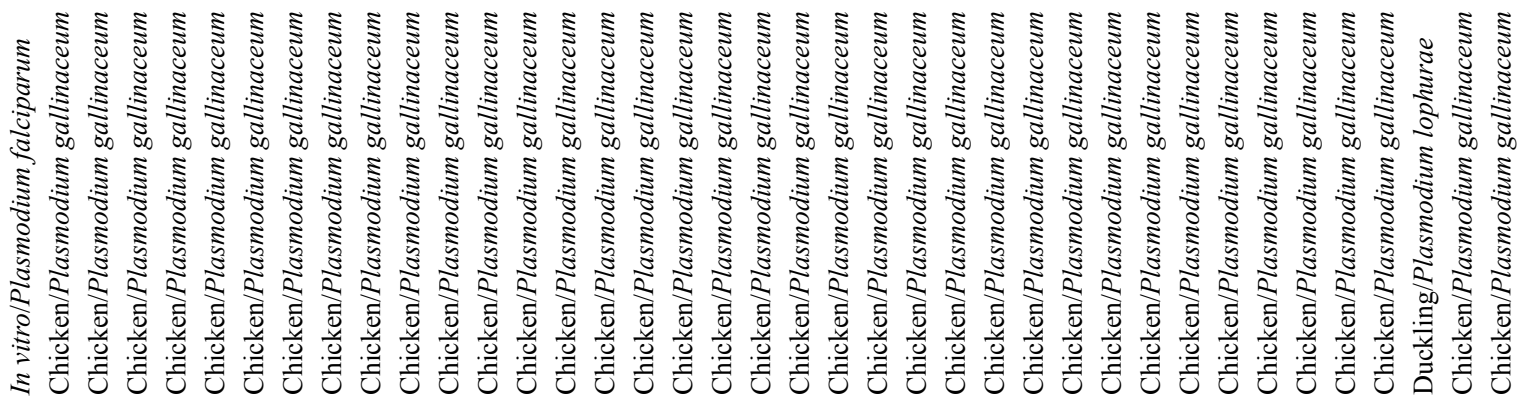

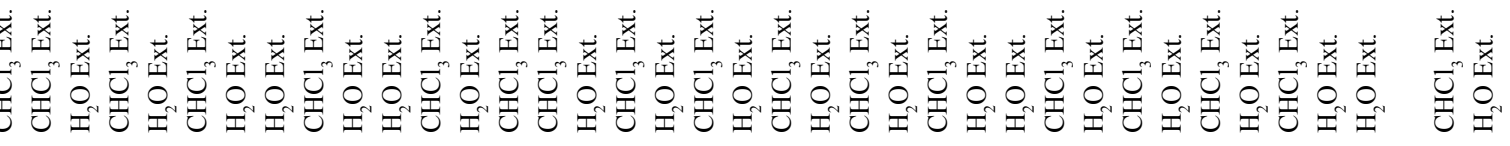

割泀

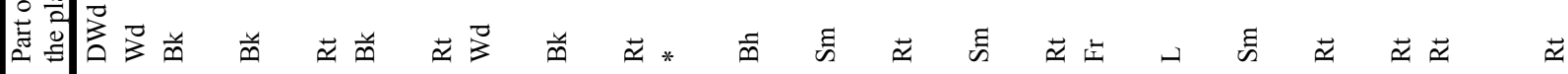

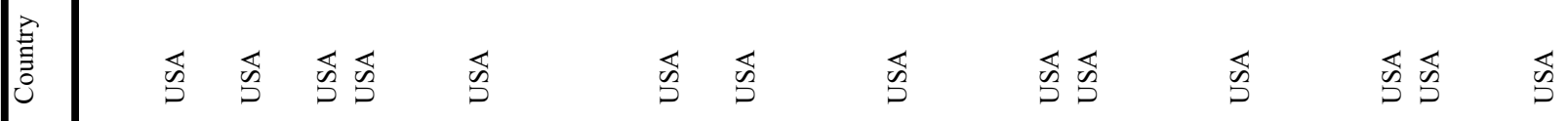




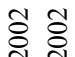

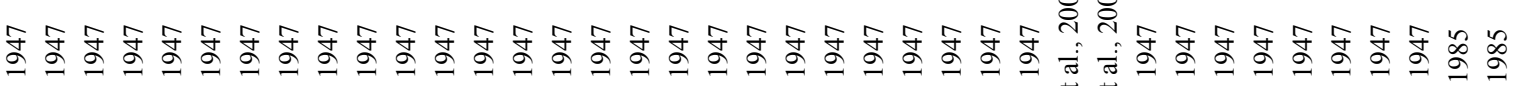

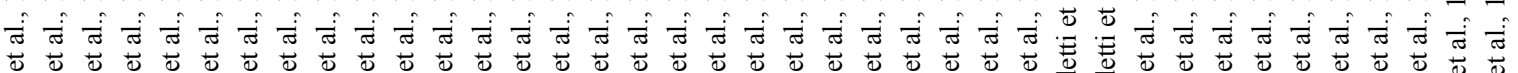

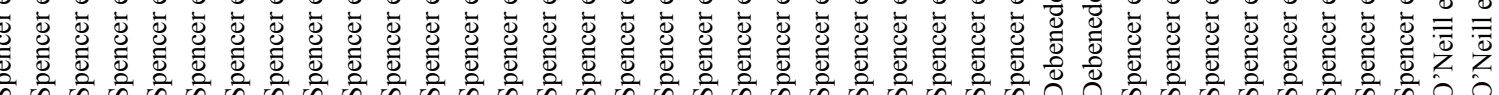

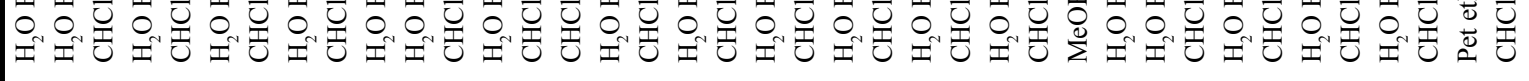

눙요

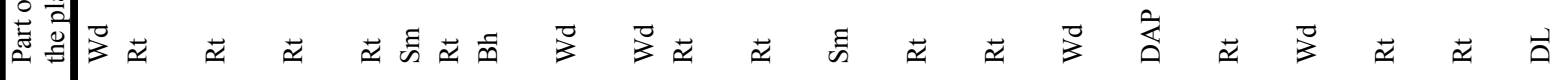

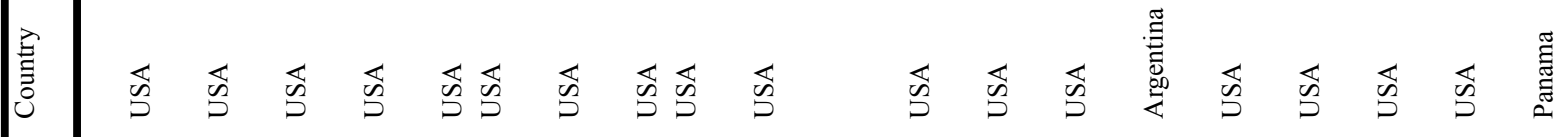

\begin{tabular}{|c|c|c|c|c|c|c|c|c|c|c|c|c|c|c|c|c|}
\hline 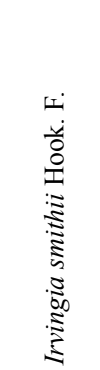 & 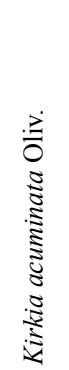 & 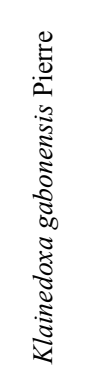 & 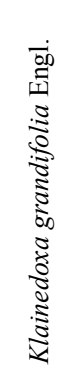 & 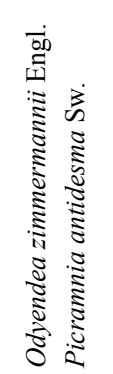 & 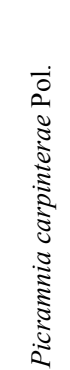 & 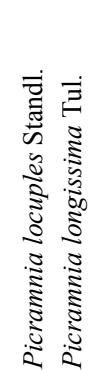 & 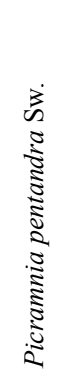 & 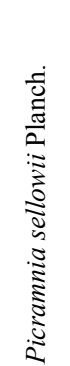 & 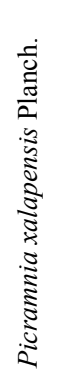 & 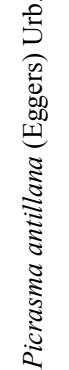 & 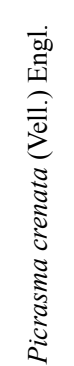 & 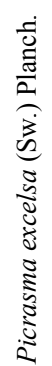 & 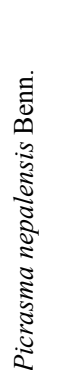 & 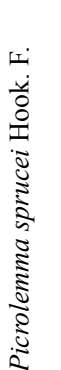 & 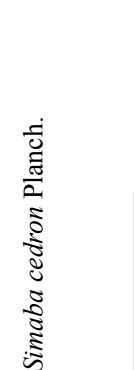 & \\
\hline & & & & & & & & & & & & & & & $\begin{array}{l}\text { acogon. } \\
\text { acogon. } \\
\text { anc2009 }\end{array}$ & 189 \\
\hline
\end{tabular}




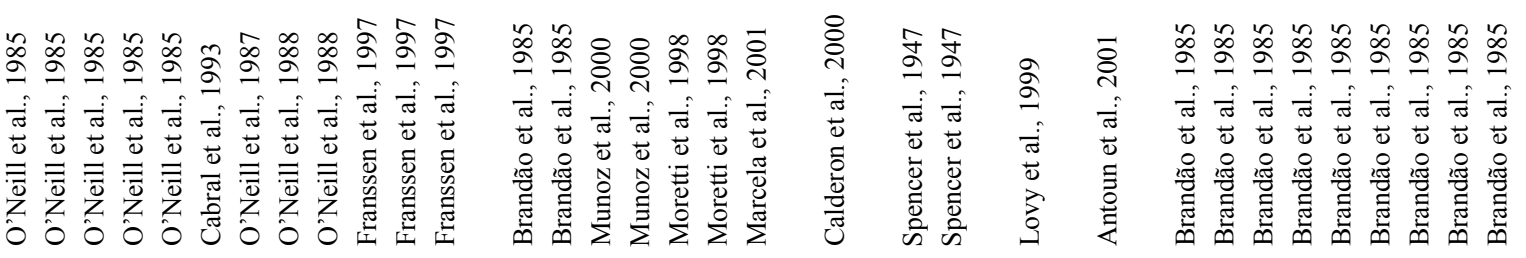

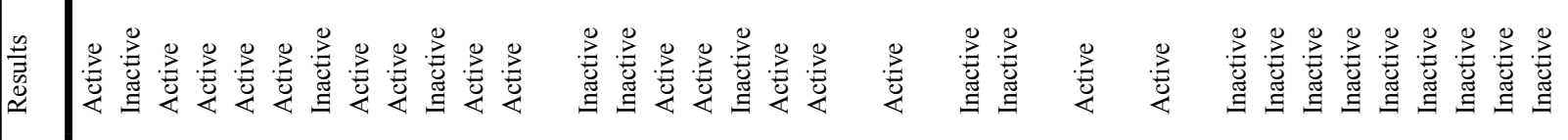
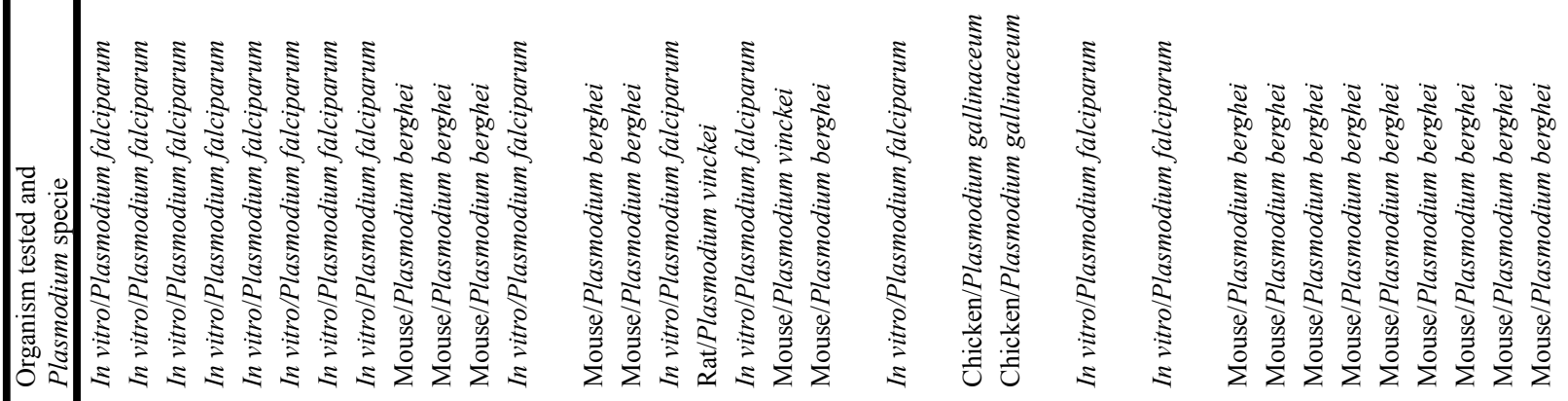

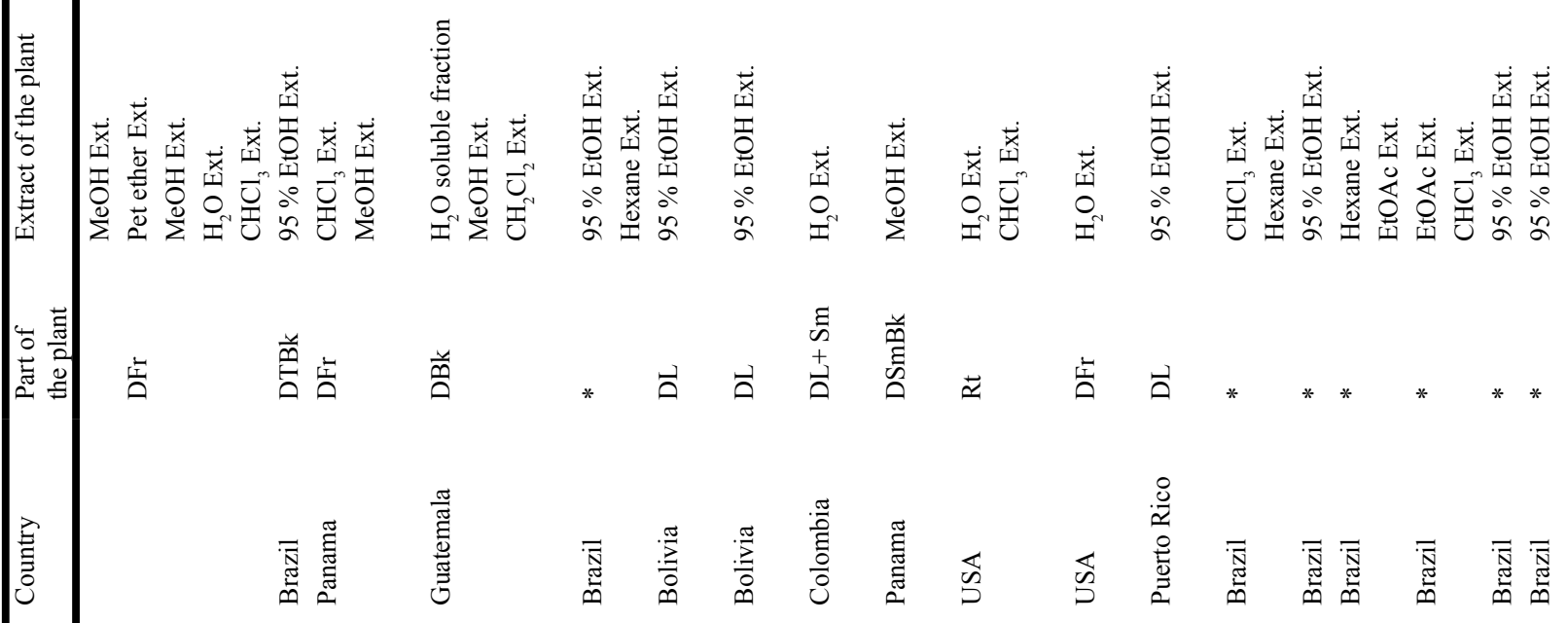

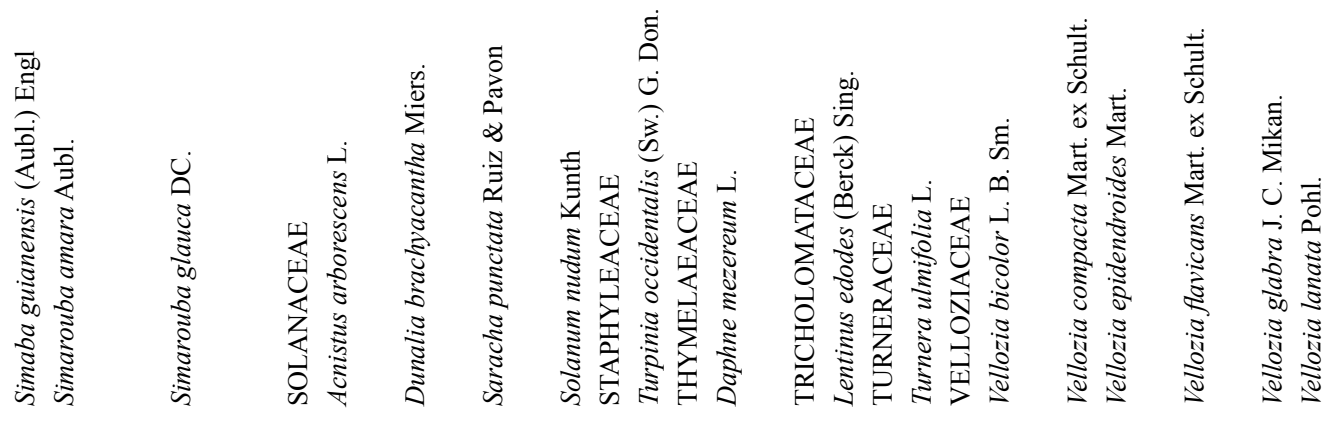




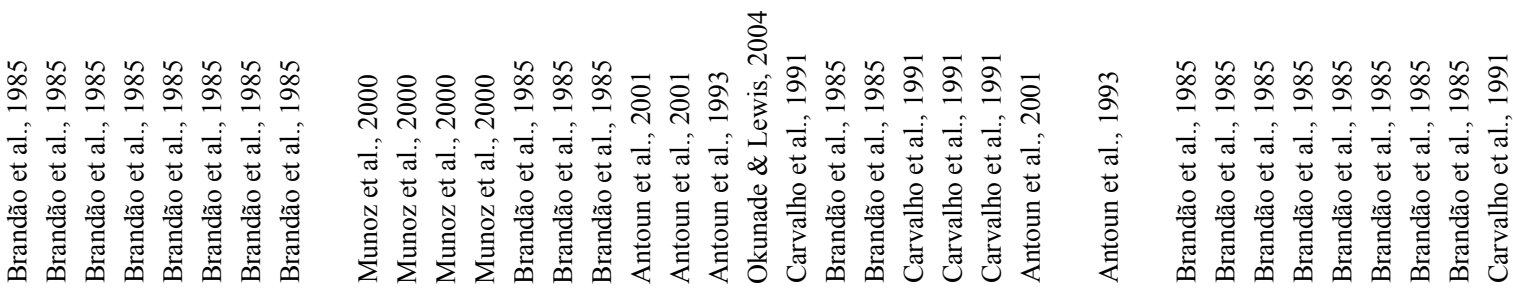

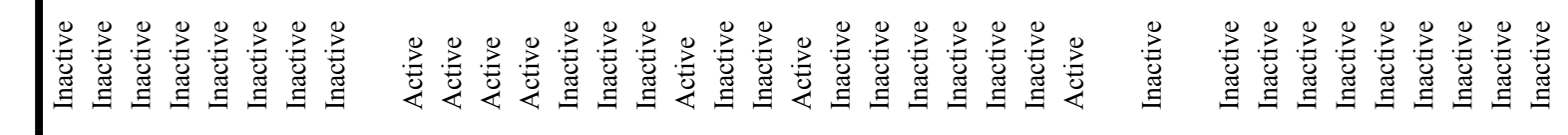

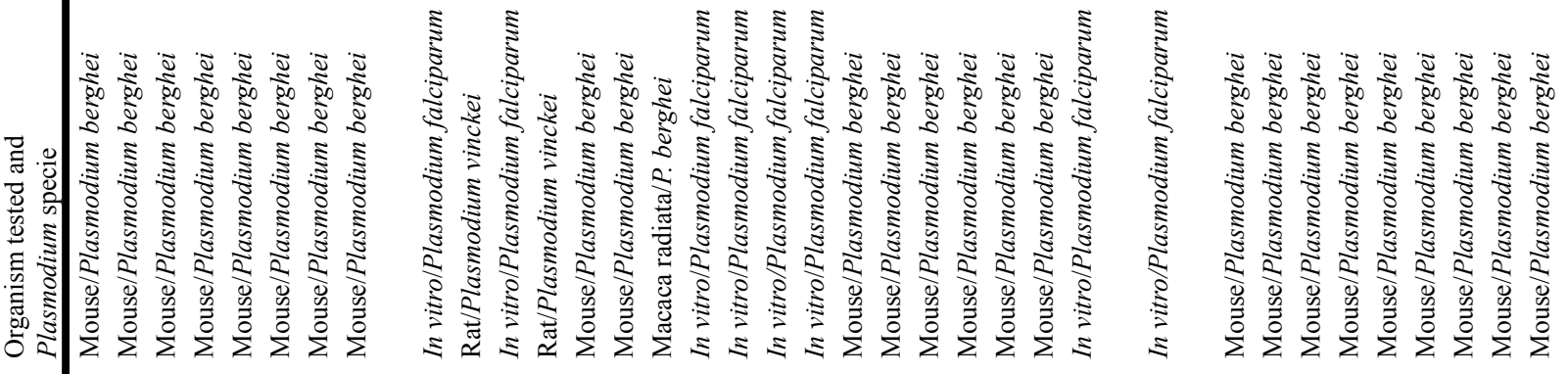

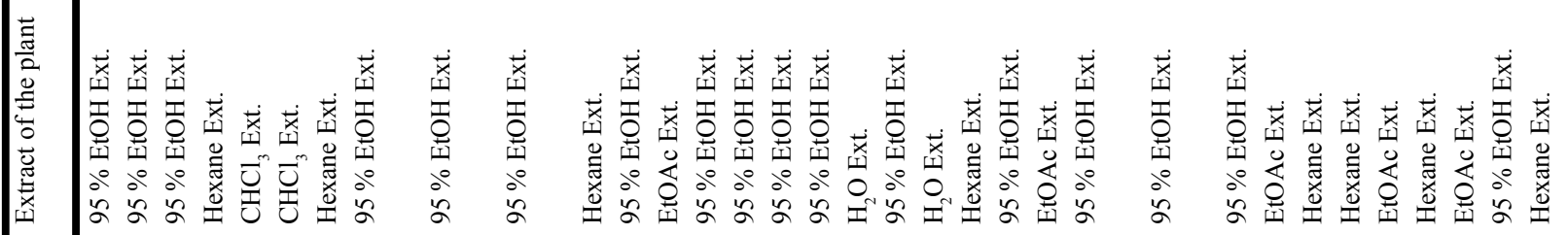

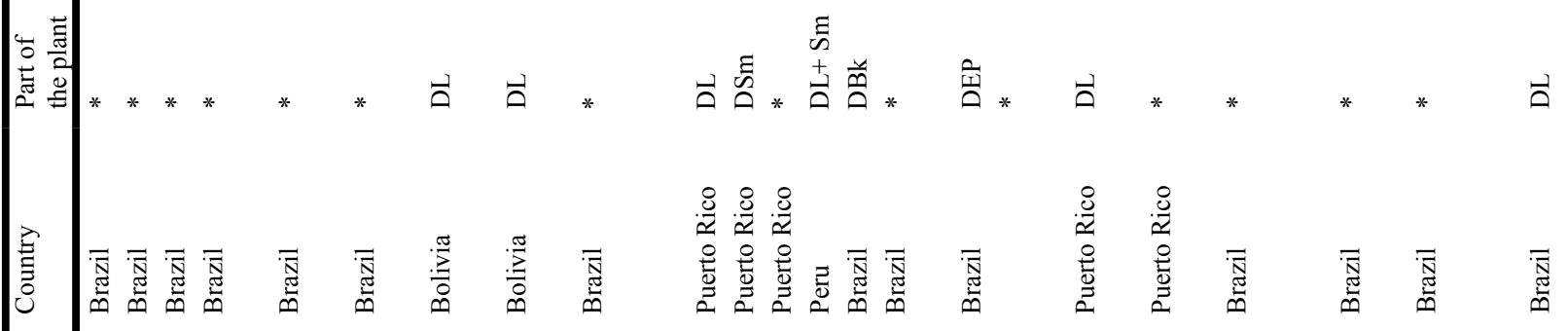

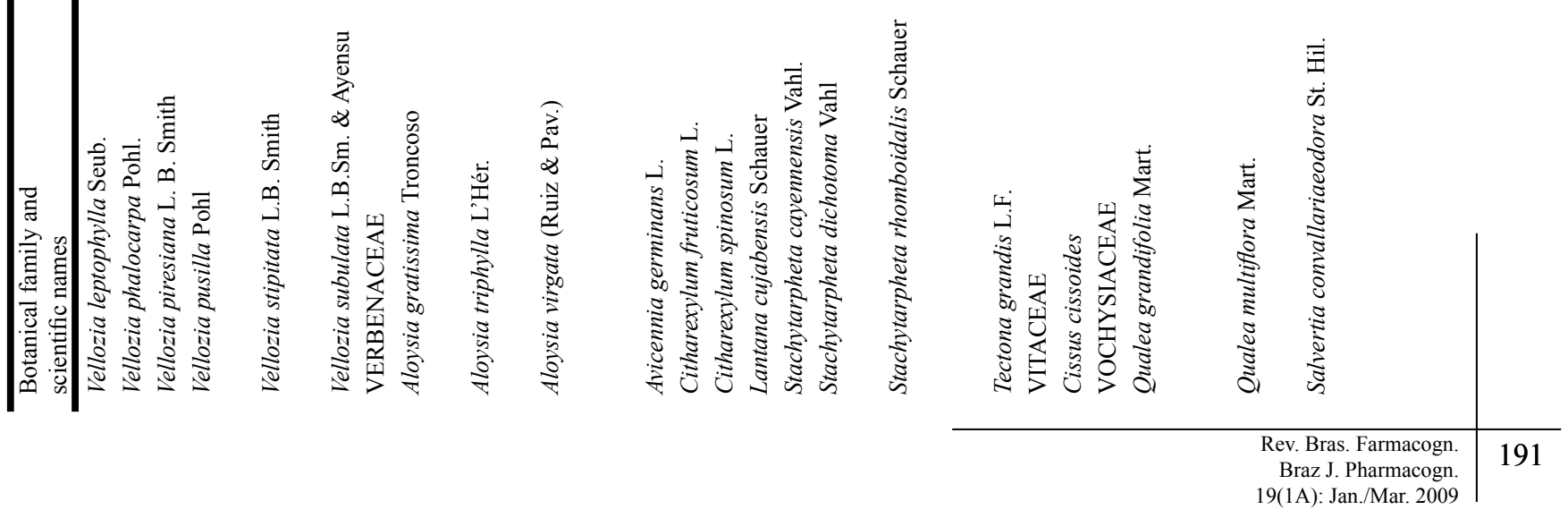

